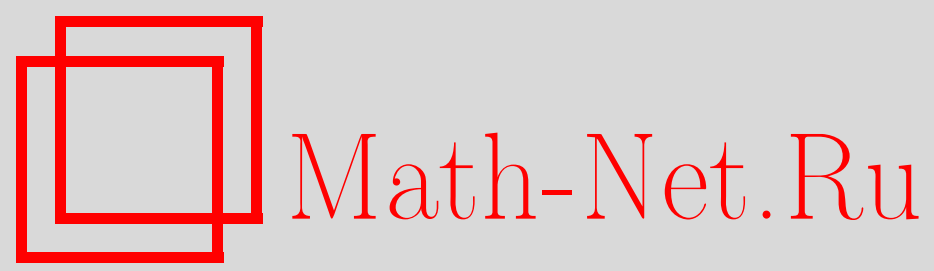

Ю. В. Немировский, А. П. Янковский, Упругопластический динамический изгиб слоисто-волокнистых пластин при действии нагрузок взрывного типа, Becmн. Сам. гос. техн. ун-та. Сер. Физ.-мат. науки, 2004, выпуск 30, 22-40

DOI: https://doi.org/10.14498/vsgtu305

Использование Общероссийского математического портала Math-Net.Ru подразумевает, что вы прочитали и согласны с пользовательским соглашением

http://www.mathnet.ru/rus/agreement

Параметры загрузки:

IP : 18.209 .158 .208

26 апреля 2023 г., 02:54:47 


\section{УПРУГОПЛАСТИЧЕСКИЙ ДИНАМИЧЕСКИЙ ИЗГИБ СЛОИСТО-ВОЛОКНИСТЫХ ПЛАСТИН ПРИ ДЕЙСТВИИ НАГРУЗОК ВЗРЫВНОГО ТИПА}

Сформулирована задача упругопластического динамического изгиба слоисто-волокнистых пластин. Разработан оригинальный метод численного интегрирования поставленной задачи, основанный на обобщении методов Рунге-Кутта. Проведены конкретные расчеты по определению предельного динамического состояния прямоугольных удлиненных изотропных, армированных однослойных и трехслойных пластин постоянной и переменной толщины и исследовано динамическое поведение таких конструкций до появления вторичной пластичности в фазах композиции.

Введение. Конструктивные элементы в виде тонких пластин составляют основу многих защитных ограждений и ответственных элементов судостроительной, машиностроительной и авиационной техники, объектов стройиндустрии. При воздействии взрывных нагрузок высокой интенсивности их повреждаемость определяет возможность дальнейшего функционирования рассматриваемых объектов. Поэтому проблема динамического расчета таких элементов конструкций является одной из важнейших в механике деформируемого твердого тела. Большинство существующих на данный момент решений основано на модели идеального жесткопластического тела. Их обзор и анализ приведены в [1, 2 и др.]. Существенным недостатком таких решений является то, что мерой повреждаемости жесткопластической конструкции служит остаточный прогиб, по которому нельзя судить о степени разрушения материала изделия. Кроме того, в [3] авторами на примере упругопластической динамики стержней показано: приближение реальной диаграммы деформирования материала конструкции к жесткопластической схеме приводит к значительному (иногда в разы) завышению величины остаточного прогиба и уменьшению периода времени, за который достигается максимум модуля прогиба конструкции.

Проблема упругопластического изгиба при оценке повреждаемости до сих пор в основном рассматривалась применительно к однородным изотропным балкам и плитам $[4,5]$. Особенностью этих исследований является то, что в них изучалась неупругая динамика конструкций лишь на начальном этапе деформирования - первичная пластичность и последующая разгрузка в течение времени, равном 1-3 периодам собственных упругих колебаний конструкции. Однако в [3] показано, что вторичная и третичная пластичность, а также начальное разрушение могут появиться и после нескольких десятков периодов собственных упругих колебаний. Искусственное же занижение характерного периода времени, в течение которого изучается процесс колебаний, может приводить к неоправданному завышению амплитуд и интенсивностей взрывных нагрузок, выдерживаемых конструкцией [3].

В последние десятилетия в инженерной практике в качестве эффективных защитных элементов используются слоистые плиты, армированные высокопрочными и высокомодульными волокнами. Исследование их неупругого деформирования находится пока в зачаточном состоянии [6]. Целью настоящей работы является разработка общего подхода к анализу динамического упругопластического деформирования слоисто-волокнистых пластин при произвольных структурах армирования и метода интегрирования соответствующей начально-краевой задачи.

Постановка задачи упругопластической динамики слоисто-волокнистых пластин. Будем исследовать упругопластический поперечный изгиб кирхгофовской пластины переменной толщины $2 H$, состоящей из нечетного числа армированных слоев, расположенных симметрично относительно среднего слоя: именно, к среднему слою с обеих сторон примыкают два слоя одинаковой толщины с одинаковыми структурами армирования и фазовыми материалами; к внешним поверхностям этих слоев примыкают еще два одинаковых слоя и т.д. Предполагается, что по толщине каждый слой имеет регулярную и квазиоднородную структуру, скольжение слоев друг по другу невозможно, так как слои склеены или спаяны друг с другом по поверхностям соприкосновения и при деформации работают совместно. Тепловое воздействие не учитывается, прогибы считаются малыми.

Для описания нелинейно-упругого или неупругого поведения фазовых материалов используются соотношения теории упругопластических деформаций $[7,8]$. Обоснованность использования этих соотношений можно подкрепить результатами исследований, проведенных в [3], где на примерах неупругой динамики стержней показано, что при нагрузках взрывного типа, не 
вызывающих появления вторичной или третичной пластичности, конструкция колеблется по формам, близким к первой собственной форме упругих колебаний. Возникающие при этом пластические деформации малы по величине и сопоставимы с предельными упругими деформациями. Ограничиваясь исследованием динамики пластин лишь до появления вторичной или третичной пластичности, можно предположить, что при взрывных нагрузках пластины, как и стержни, будут колебаться практически неотличимо от первой собственной формы упругих колебаний, т.е. путь деформирования точек конструкции будет мало отличаться от простого и пластические деформации будут сопоставимы по модулю с предельными упругими деформациями фаз композиции, в силу чего можно обоснованно использовать деформационную теорию пластичности [9]. Кроме того, в [3] продемонстрировано, что возможно возникновение начального разрушения колеблющейся конструкции еще до появления третичной пластичности, поэтому деформационная теория может оказаться пригодной и для изучения предельного динамического состояния конструкций из некоторых типов композитных материалов, особенно для металлических пластин, армированных упруго-хрупкими волокнами (композиции типа $\mathrm{Al}-\mathrm{B}$, $\mathrm{Al}-\mathrm{C}, \mathrm{Mg}-\mathrm{B}, \mathrm{Mg}-\mathrm{C}, \mathrm{Al}-\mathrm{W}, \mathrm{Mg}-\mathrm{W}, \mathrm{Ni}-\mathrm{W}, \mathrm{Ni}-\mathrm{C}, \mathrm{Cu}-\mathrm{C}$ и др.) и в которых пластические деформации в связующей матрице, возникающие к моменту появления начального разрушения таких волокон, будут по модулю, как правило, всего в 1,5-4 раза больше предельной упругой деформации связующего (т.е. сопоставимы с последней), так как предельная деформация волокон в таких композициях всего 2,5-5 раз больше предельной упругой деформации связующего [10].

Пластина рассматривается в прямоугольной декартовой системе координат $x_{1} x_{2} z$; плоскость $x_{1} x_{2}$ совмещена со срединной плоскостью центрального слоя (она же срединная плоскость пластины) до изгиба, а ось $z$ перпендикулярна этой плоскости. Центральный слой будем называть «нулевым»; выше него $(z>0)$ последовательно располагаются слои с номерами $m=1,2, \ldots, M$, ниже $(z<0)$ - слои с номерами $m=M+1, M+2, \ldots, 2 M$ (всего $2 M+1$ слоев, причем $M$-й слой является верхним, а $2 M$-ый слой - нижним). Каждый $m$-ый слой армирован $N^{(m)}$ семействами волокон (возможно, различной физической природы), которые уложены в плоскостях, параллельных плоскости $x_{1} x_{2}$ (плоское армирование), или на поверхностях, расстояния между которыми по оси $z$ изменяются пропорционально изменению толщины слоя (пространственное армирование).

Обозначим через $H_{m}\left(x_{1}, x_{2}\right) \geq 0$ аппликату границы между $m$-ным и $(m+1)$-ым слоями $\left(0 \leq m \leq M, H_{M}=H\right)$, тогда $z=-H_{m} \leq 0$ будет задавать границу между $(m+M)$-ым и $(m+M+1)$-ым слоями $(1 \leq m \leq M)$, а $z=-H_{0} \leq 0$ - границу между центральным слоем и соседним нижним $(M+1)$-ым слоем, при этом $2 H_{0} \geq 0$ - толщина среднего слоя, $H_{m}-H_{m-1} \geq 0$ — толщина $m$-го и $(m+M)$-го слоев $(1 \leq m \leq M)$.

Распределенная нагрузка $p\left(x_{1}, x_{2}, t\right)$ действует в направлении $z$, поэтому при сделанных выше предположениях о структуре пластины в случае малых прогибов $w\left(x_{1}, x_{2}, t\right)$ будет реализоваться поперечный изгиб конструкции. Уравнения динамического изгиба такой пластины без учета инерции вращения согласно принципу Даламбера имеют вид

$$
F_{1,1}+F_{2,2}+p=R\left(x_{1}, x_{2}\right) w_{t t}\left(x_{1}, x_{2}, t\right), M_{i 1,{ }_{1}}+M_{i 2},_{2}=F_{i}, \quad i=1,2,
$$

где

$$
\begin{aligned}
& M_{i j}=\int_{-H}^{H} \sigma_{i j} z d z=2 \sum_{m=0}^{M} \int_{H_{m-1}}^{H_{m}} \sigma_{i j}^{(m)} z d z \quad(i, j=1,2), \quad R=\int_{-H}^{H} \rho\left(x_{1}, x_{2}, z\right) d z=2 \sum_{m=0}^{M} \int_{H_{m-1}}^{H_{m}} \rho^{(m)} d z \quad\left(H_{-1} \equiv 0\right), \\
& \rho^{(m)}=a^{(m)} \rho_{\mathrm{c}}^{(m)}+\sum_{k} \rho_{k}^{(m)} \omega_{k}^{(m)}\left(x_{1}, x_{2}\right), \quad a^{(m)}\left(x_{1}, x_{2}\right)=1-\sum_{k} \omega_{k}^{(m)}\left(x_{1}, x_{2}\right) ;
\end{aligned}
$$

$M_{i j}, F_{i}$ - внутренние силовые факторы (моменты и перерезывающие силы соответственно); $\sigma_{i j}^{(m)}$ - осредненные напряжения в композиции $m$-ного слоя; $\rho_{\mathrm{c}}^{(m)}, \rho_{k}^{(m)}$ - объемные плотности материалов связующего и арматуры $k$-того семейства $m$-ного слоя соответственно; $\omega_{k}^{(m)}-$ интенсивность (плотность) армирования $m$-того слоя волокнами $k$-того семейства $\left(1 \leq k \leq N^{(m)}\right)$. Суммирование здесь и далее производится по указанному индексу от 1 до $N^{(m)}$, если не проставлены пределы; нижний индекс после запятой означает частное дифференцирование по пространственной переменной $x_{i}$ или времени $t$ соответственно; верхний индекс в скобках озна- 
чает номер армированного слоя (если некоторые соотношения формально выполняются для всех слоев, то для упрощения записи далее будем опускать верхний индекс в скобках).

Осредненные напряжения $\sigma_{i j}^{(m)}$ в (2) выразим через напряжения в фазовых материалах $m$ ного слоя (используется структурная модель армированного слоя из [11])

$$
\sigma_{i j}^{(m)}=a^{(m)} \sigma_{\mathrm{c} i j}^{(m)}+\sum_{k} \sigma_{k}^{(m)} \omega_{k}^{(m)} l_{k i}^{(m)} l_{k j}^{(m)} \quad(i, j=1,2), \quad l_{k 1}^{(m)}=\cos \psi_{k}^{(m)}, \quad l_{k 2}^{(m)}=\sin \psi_{k}^{(m)},
$$

где $\sigma_{\text {сі } i j}^{(m)}, \sigma_{k}^{(m)}$ - напряжения в связующем и арматуре $k$-того семейства $m$-ного слоя соответственно; $\psi_{k}^{(m)}$ - угол армирования $m$-того слоя волокнами $k$-того семейства, отсчитываемый от направления $x_{1}$.

Предположим, что диаграммы растяжения и сжатия всех фазовых материалов совпадают и имеют линейное упрочнение. Тогда связь между напряжением $\sigma_{k}$ и деформацией $\varepsilon_{k}$ арматуры $k$-того семейства до начала разгрузки имеет вид $[7,11]$

$$
\sigma_{k}=\left\{\begin{array}{l}
E_{k} \varepsilon_{k},\left|\varepsilon_{k}\right| \leq \varepsilon_{\mathrm{T} k}=\sigma_{\mathrm{T} k} / E_{k} ; \\
\operatorname{sign}\left(\varepsilon_{k}\right) \sigma_{\mathrm{T} k}+E_{\mathrm{T} k}\left(\varepsilon_{k}-\operatorname{sign}\left(\varepsilon_{k}\right) \varepsilon_{\mathrm{T} k}\right), \varepsilon_{\mathrm{T} k}<\left|\varepsilon_{k}\right| \leq \varepsilon_{\mathrm{B} k},
\end{array}\right.
$$

где $\sigma_{\text {тk }} \equiv \sigma_{\mathrm{T} k}^{(m)}$ - предел текучести материала волокон $k$-того семейства в $m$-ном слое; $E_{k} \equiv E_{k}^{(m)}, E_{\mathrm{\tau} k} \equiv E_{\mathrm{\tau} k}^{(m)}-$ модули упругости и упрочнения материала волокон $k$-того семейства в $m$-ном слое; $\varepsilon_{\mathrm{T} k} \equiv \varepsilon_{\mathrm{T} k}^{(m)}, \varepsilon_{\mathrm{B} k} \equiv \varepsilon_{\mathrm{B} k}^{(m)}-$ деформации, соответствующие пределу текучести и временному сопротивлению $\sigma_{\mathrm{B} k}^{(m)}$ материала волокон $k$-того семейства в $m$-ном слое.

Связь между деформациями пластины $\varepsilon_{i j}$ и деформациями $\varepsilon_{k}$ волокон $k$-того семейства в рамках используемой структурной модели определяется соотношениями [11]

$$
\varepsilon_{k}=\varepsilon_{11} \cos ^{2} \psi_{k}+\varepsilon_{22} \sin ^{2} \psi_{k}+\varepsilon_{12} \sin 2 \psi_{k}, k=1,2, \ldots, N,
$$

где в силу кинематических гипотез Кирхгофа

$$
\varepsilon_{i j}=-z w_{i j}, i, j=1,2 \quad(|z| \leq H) .
$$

Из (4)-(6) следует выражение для напряжений в волокнах $k$-того семейства в $m$-ном слое (до начала первичной разгрузки)

$$
\sigma_{k}^{(m)}=\left\{\begin{array}{l}
E_{k}^{(m)} e_{k}^{(m)} z, \quad H_{m-1}<z \leq h_{k}^{(m)} ; \\
\operatorname{sign}\left(e_{k}^{(m)} z\right) \sigma_{\mathrm{\tau} k}^{(m)}+E_{\mathrm{\tau} k}^{(m)}\left[e_{k}^{(m)} z-\operatorname{sign}\left(e_{k}^{(m)} z\right) \varepsilon_{\mathrm{\tau} k}^{(m)}\right], \quad h_{k}^{(m)}<z \leq H_{m},
\end{array}\right.
$$

где

$$
\begin{gathered}
h_{k}^{(m)}=\left\{\begin{array}{l}
H_{m} \text { при } h_{* k}^{(m)} \geq H_{m} ; \\
h_{* k}^{(m)} \text { при } H_{m-1}<h_{* k}^{(m)}<H_{m} ; \quad h_{* k}^{(m)}=\frac{\varepsilon_{\mathrm{\tau} k}^{(m)}}{\left|e_{k}^{(m)}\right|}=\frac{\sigma_{\mathrm{\tau k}}^{(m)}}{E_{k}^{(m)}\left|e_{k}^{(m)}\right|} ; \\
H_{m-1} \text { при } h_{* k}^{(m)} \leq H_{m-1},
\end{array}\right. \\
e_{k}^{(m)}=-w_{, 11} \cos ^{2} \psi_{k}^{(m)}-w,_{22} \sin ^{2} \psi_{k}^{(m)}-w_{, 12} \sin 2 \psi_{k}^{(m)}, \quad 1 \leq k \leq N^{(m)}, \quad 0 \leq m \leq M ;
\end{gathered}
$$

$h_{k}^{(m)}>0$ аппликата границы между упругой и неупругой зонами в арматуре $k$-того семейства в $m$-ном слое (т.е. при $H_{m-1}<|z| \leq h_{k}^{(m)}$ волокна $k$-того семейства в $m$-ном и $(m+M)$-ном слоях ведут себя упруго, а при $h_{k}^{(m)}<|z| \leq H_{m}-$ неупруго); $e_{k}^{(m)}$ - параметр искривления срединной плоскости пластины в направлении армирования волокном $k$-того семейства в $m$-ном слое.

Линейно-упругое поведение материала связующего каждого слоя согласно статической гипотезе Кирхгофа определяется законом Гука

$$
\sigma_{\mathrm{c} i i}=E a_{1}\left(\varepsilon_{i i}+v \varepsilon_{j j}\right), \sigma_{\mathrm{c} i j}=E a_{2} \varepsilon_{i j}, j=3-i, \quad i=1,2,
$$

а интенсивность деформаций $\varepsilon_{0 u}$ при этом равна $[7,8]$

$$
\varepsilon_{0 u}=\frac{2}{\sqrt{3}} \sqrt{\frac{1-v+v^{2}}{3(1-v)^{2}}\left(\varepsilon_{11}^{2}-\frac{1-4 v+v^{2}}{1-v+v^{2}} \varepsilon_{11} \varepsilon_{22}+\varepsilon_{22}^{2}\right)+\varepsilon_{12}^{2}},
$$

где

$$
a_{1}=1 /\left(1-v^{2}\right), \quad a_{2}=1 /(1+v) ;
$$


$E \equiv E^{(m)}, v \equiv v^{(m)}$ - модуль упругости и коэффициент Пуассона связующего $m$-го слоя.

Нелинейно-упругое и неупругое поведение материала связующего определяется основными соотношениями теории упругопластических деформаций $[7,8]$, упрощенными допущением о несжимаемости материала (не меняя существа дела, учет сжимаемости при неупругом деформировании связующих материалов слоев изгибаемой пластины связан со значительными техническими трудностями и для армированной конструкции практически не влияет на результаты расчетов [12]). В случае использования диаграммы деформирования с линейным упрочнением связь между напряжениями $\sigma_{\text {cij }}$ и деформациями $\varepsilon_{i j}$ за пределами линейной упругости при отсутствии дилатации имеет вид:

$$
\sigma_{\mathrm{c} i i}=\frac{2\left[\sigma_{\mathrm{T}}+E_{*}\left(\varepsilon_{u}-\varepsilon_{*}\right)\right]}{3 \varepsilon_{u}}\left(2 \varepsilon_{i i}+\varepsilon_{j j}\right), \quad \sigma_{\mathrm{c} i j}=\frac{2\left[\sigma_{\mathrm{T}}+E_{*}\left(\varepsilon_{u}-\varepsilon_{*}\right)\right]}{3 \varepsilon_{u}} \varepsilon_{i j}, j=3-i, i=1,2,
$$

где $\sigma_{\mathrm{T}} \equiv \sigma_{\mathrm{T}}^{(m)}, E_{*} \equiv E_{*}^{(m)}-$ предел текучести и модуль упрочнения материала связующего $m$-го слоя, известные из диаграммы деформирования [7]; $\varepsilon_{*}-$ значение интенсивности деформаций, соответствующее $\sigma_{\text {т }}$ на диаграмме деформирования; интенсивность деформаций $\varepsilon_{u}$ в предположении о несжимаемости материала связующего каждого слоя имеет выражение [8]

$$
\varepsilon_{u}^{2}=4\left(\varepsilon_{11}^{2}+\varepsilon_{11} \varepsilon_{22}+\varepsilon_{22}^{2}+\varepsilon_{12}^{2}\right) / 3 .
$$

Введем в рассмотрение положительные величины $e_{0 u}, e_{u}$, составленные из параметров искривления $w_{i_{i j}}$ срединной плоскости пластины таким же образом, как и интенсивности $\varepsilon_{0 u}, \varepsilon_{u}$ (11), (14) соответственно,

$$
\begin{gathered}
e_{0 u}=\frac{2}{\sqrt{3}} \sqrt{\frac{1-v+v^{2}}{3(1-v)^{2}}\left(w_{, 11}^{2}-\frac{1-4 v+v^{2}}{1-v+v^{2}} w_{, 11} w_{, 22}+w_{, 22}^{2}\right)+w_{, 12}^{2}} ; \\
e_{u}^{2}=4\left(w_{, 11}^{2}+w_{, 11} w_{, 22}+w_{, 22}^{2}+w_{, 12}^{2}\right) / 3,
\end{gathered}
$$

тогда из соотношений (6), (11), (14)-(16) следует

$$
\varepsilon_{0 u}^{(m)}=|z| e_{0 u}^{(m)}\left(x_{1}, x_{2}, t\right), \quad \varepsilon_{u}=|z| e_{u}\left(x_{1}, x_{2}, t\right) .
$$

Если $h^{(m)}$ - аппликата границы между упругой и неупругой зонами в связующем $m$-ного слоя, то интенсивность напряжений

$$
\sigma_{u}^{(m)} \leq \sigma_{\mathrm{T}}^{(m)}\left(H_{m-1}<z \leq h^{(m)}\right), \quad \sigma_{u}^{(m)}>\sigma_{\mathrm{T}}^{(m)} \quad\left(h^{(m)}<z \leq H_{m}\right) .
$$

В упругой зоне связующего $m$-го слоя интенсивность напряжений $[7,8]$ :

$$
\sigma_{u}^{(m)}=3 G_{\mathrm{c}}^{(m)} \varepsilon_{0 u}^{(m)}=3 G_{\mathrm{c}}^{(m)}|z| e_{0 u}^{(m)}, G_{\mathrm{c}}^{(m)}=0,5 E^{(m)} /\left(1+v^{(m)}\right),
$$

где $G_{\mathrm{c}}^{(m)}$ - модуль сдвига связующего. На границах $z=h^{(m)}$ между упругой и неупругой зонами в связующем $\sigma_{u}^{(m)}=\sigma_{\mathrm{T}}^{(m)}$, поэтому из (19) следует

$$
h_{*}^{(m)}=\sigma_{\mathrm{T}}^{(m)} /\left(3 G_{\mathrm{c}}^{(m)} e_{0 u}^{(m)}\right),
$$

откуда

$$
h^{(m)}=\left\{\begin{array}{l}
H_{m} \text { при } h_{*}^{(m)} \geq H_{m} ; \\
h_{*}^{(m)} \text { при } H_{m-1}<h_{*}^{(m)}<H_{m} ; \\
H_{m-1} \text { при } h_{*}^{(m)} \leq H_{m-1}, m=0,1, \ldots, M, H_{-1} \equiv 0 .
\end{array}\right.
$$
гиба.

Соотношения (15), (20), (21) определяют функцию $h^{(m)}$ через вторые производные от про-

Окончательно напряжения $\sigma_{\text {сij }}^{(m)}$ в связующем $m$-ного слоя с учетом выражений (6), (10), (13), (17) задаются соотношениями

$$
\begin{gathered}
\sigma_{\mathrm{c} i i}^{(m)}=-z E^{(m)} a_{1}^{(m)}\left(w_{,_{i i}}+v^{(m)} w_{, j j}\right), \sigma_{\mathrm{c} i j}^{(m)}=-z E^{(m)} a_{2}^{(m)} w_{i j} \quad\left(H_{m-1}<|z| \leq h^{(m)}\right) ; \\
\sigma_{\mathrm{c} i i}^{(m)}=-2\left[z E_{*}^{(m)}+\operatorname{sign}(z)\left(\sigma_{\mathrm{T}}^{(m)}-E_{*}^{(m)} \varepsilon_{*}^{(m)}\right) / e_{u}\right]\left(2 w_{i i}+w_{, j j}\right) / 3 ; \\
\sigma_{\mathrm{c} i j}^{(m)}=-2\left[z E_{*}^{(m)}+\operatorname{sign}(z)\left(\sigma_{\mathrm{T}}^{(m)}-E_{*}^{(m)} \varepsilon_{*}^{(m)}\right) / e_{u}\right] w_{, i j} / 3 \quad\left(h^{(m)}<|z| \leq H_{m}\right), \quad j=3-i, \quad i=1,2,
\end{gathered}
$$

где величины $a_{1}^{(m)}, a_{2}^{(m)}$ определены в (12). 
Подставляя в выражения (2) соотношения (3) и учитывая представления (7), (8), (21)—(23), получим выражения для моментов до начала разгрузки через прогиб слоисто-волокнистой пластины:

$$
M_{i j}\left(x_{1}, x_{2}, t\right)=-\frac{2}{3}\left(\sum_{s=1}^{2} \sum_{l=1}^{2} C_{i j s l}\left(x_{1}, x_{2} ; w\right) w,_{s l}\left(x_{1}, x_{2}, t\right)-D_{i j}\left(x_{1}, x_{2} ; w\right)\right), i, j=1,2,
$$

где

$$
\begin{aligned}
& C_{i i i i}=\sum_{m=0}^{M}\left\{\sum_{k} \omega_{k}^{(m)}\left(l_{k i}^{(m)}\right)^{4}\left[E_{k}^{(m)}\left(\left(h_{k}^{(m)}\right)^{3}-H_{m-1}^{3}\right)+E_{\mathrm{r} k}^{(m)}\left(H_{m}^{3}-\left(h_{k}^{(m)}\right)^{3}\right)\right]+a^{(m)}\left[a_{1}^{(m)} E^{(m)} \times\right.\right. \\
& \left.\left.\times\left(\left(h^{(m)}\right)^{3}-H_{m-1}^{3}\right)+4\left[E_{*}^{(m)}\left(H_{m}^{3}-\left(h^{(m)}\right)^{3}\right) / 3+\left(H_{m}^{2}-\left(h^{(m)}\right)^{2}\right)\left(\sigma_{\mathrm{T}}^{(m)}-E_{*}^{(m)} \varepsilon_{*}^{(m)}\right) / e_{u}\right]\right]\right\}, C_{i j j j}= \\
& =\sum_{m=0}^{M}\left\{\sum_{k} \omega_{k}^{(m)}\left(l_{k i}^{(m)}\right)^{2}\left(l_{k j}^{(m)}\right)^{2}\left[E_{k}^{(m)}\left(\left(h_{k}^{(m)}\right)^{3}-H_{m-1}^{3}\right)+E_{\mathrm{T} k}^{(m)}\left(H_{m}^{3}-\left(h_{k}^{(m)}\right)^{3}\right)\right]+a^{(m)}\left[a_{1}^{(m)} E^{(m)} v^{(m)} \times\right.\right. \\
& \left.\left.\times\left(\left(h^{(m)}\right)^{3}-H_{m-1}^{3}\right)+2\left[E_{*}^{(m)}\left(H_{m}^{3}-\left(h^{(m)}\right)^{3}\right) / 3+\left(H_{m}^{2}-\left(h^{(m)}\right)^{2}\right)\left(\sigma_{\mathrm{T}}^{(m)}-E_{*}^{(m)} \varepsilon_{*}^{(m)}\right) / e_{u}\right]\right]\right\}, C_{i i i j}= \\
& =\sum_{m=0}^{M} \sum_{k} \omega_{k}^{(m)}\left(l_{k i}^{(m)}\right)^{3} l_{k j}^{(m)}\left[E_{k}^{(m)}\left(\left(h_{k}^{(m)}\right)^{3}-H_{m-1}^{3}\right)+E_{\mathrm{\tau} k}^{(m)}\left(H_{m}^{3}-\left(h_{k}^{(m)}\right)^{3}\right)\right], \quad C_{i j i j}=\sum_{m=0}^{M}\left\{\sum_{k} \omega_{k}^{(m)} \times\right. \\
& \times\left(l_{k i}^{(m)}\right)^{2}\left(l_{k j}^{(m)}\right)^{2}\left[E_{k}^{(m)}\left(\left(h_{k}^{(m)}\right)^{3}-H_{m-1}^{3}\right)+E_{\mathrm{r} k}^{(m)}\left(H_{m}^{3}-\left(h_{k}^{(m)}\right)^{3}\right)\right]+a^{(m)}\left[a_{2}^{(m)} \frac{E^{(m)}}{2}\left(\left(h^{(m)}\right)^{3}-H_{m-1}^{3}\right)+\right. \\
& \left.\left.+\frac{E_{*}^{(m)}}{3}\left(H_{m}^{3}-\left(h^{(m)}\right)^{3}\right)+\left(H_{m}^{2}-\left(h^{(m)}\right)^{2}\right)\left(\sigma_{\mathrm{T}}^{(m)}-E_{*}^{(m)} \varepsilon_{*}^{(m)}\right) / e_{u}\right]\right\} \quad(j=3-i, i=1,2), a^{(m)}=1-\sum_{k} \omega_{k}^{(m)}, \\
& D_{i j}=3 \sum_{m=0}^{M} \sum_{k} \omega_{k}^{(m)} l_{k i}^{(m)} l_{k j}^{(m)} \operatorname{sign}\left(e_{k}^{(m)}\right)\left(H_{m}^{2}-\left(h_{k}^{(m)}\right)^{2}\right)\left(\sigma_{\mathrm{T} k}^{(m)}-E_{\mathrm{T} k}^{(m)} \varepsilon_{\mathrm{T} k}^{(m)}\right) \quad(i, j=1,2), H_{-1} \equiv 0 ; \\
& e_{k}^{(m)}, e_{u}, h_{k}^{(m)}, h^{(m)}, a_{1}^{(m)}, a_{2}^{(m)} \text { определены в (8), (9), (12), (16), (20), (21). }
\end{aligned}
$$

Если в точке $\left(x_{1}, x_{2}, z\right) m$-ного слоя имеет место разгрузка волокон $k$-того семейства, то напряжения в этих волокнах равны $[7,11,13]$

$$
\sigma_{k}^{(m)}=\left\{\begin{array}{l}
E_{k}^{(m)} \varepsilon_{k}^{(m)}=z E_{k}^{(m)} e_{k}^{(m)}, H_{m-1} \leq|z| \leq h_{0 k}^{(m)} ; \\
\sigma_{0 k}^{(m)}+E_{k}^{(m)}\left(\varepsilon_{k}^{(m)}-\varepsilon_{0 k}^{(m)}\right)=\sigma_{0 k}^{(m)}+z E_{k}^{(m)}\left(e_{k}^{(m)}-e_{0 k}^{(m)}\right), \quad h_{0 k}^{(m)}<|z| \leq H_{m},
\end{array}\right.
$$

где согласно второму равенству (7)

$$
\sigma_{0 k}^{(m)}=\operatorname{sign}\left(e_{0 k}^{(m)} z\right) \sigma_{\mathrm{T} k}^{(m)}+E_{\mathrm{T} k}^{(m)}\left[e_{0 k}^{(m)} z-\operatorname{sign}\left(e_{0 k}^{(m)} z\right) \varepsilon_{\mathrm{T} k}^{(m)}\right], h_{0 k}^{(m)}<|z| \leq H_{m} ;
$$

$\varepsilon_{0 k}^{(m)}, e_{0 k}^{(m)}, h_{0 k}^{(m)}$ - значения функций $\varepsilon_{k}^{(m)}, e_{k}^{(m)}, h_{k}^{(m)}$ соответственно (см. (5), (8), (9)), определенных в момент времени $t=t_{k}^{(m)}\left(x_{1}, x_{2}\right)$ начала разгрузки волокон $k$-того семейства в $m$-ном слое, который характеризуется соотношениями

$$
e_{k, t}^{(m)}\left(x_{1}, x_{2}, t_{k}^{(m)}\right)=0, \quad e_{k, t t}^{(m)}\left(x_{1}, x_{2}, t_{k}^{(m)}\right) \neq 0,
$$

в которых следует учесть (9); при этом смешанные производные $w_{\text {, } i j t t}$, появляющиеся в левой части неравенства (28), можно определить за счет дифференцирования по $x_{i}, x_{j}$ первого уравнения (1).

Если в процессе разгрузки в точке $\left(x_{1}, x_{2}\right)$ выполняются соотношения

$$
e_{k}^{(m)}=e_{0 k}^{(m)}, \operatorname{sign}\left(e_{0 k}^{(m)}\right) e_{k, t}^{(m)}>0\left(\varepsilon_{k}^{(m)}=\varepsilon_{0 k}^{(m)}, \operatorname{sign}\left(\varepsilon_{0 k}^{(m)}\right) \varepsilon_{k, t}^{(m)}>0\right),
$$

то в волокнах $k$-того семейства в $m$-ном слое вновь будет развиваться первичная пластичность и от равенств (26), (27) следует перейти к (7)-(9). Если же в процессе разгрузки рассматриваемых волокон выполняются соотношения

$$
\left|\varepsilon_{k}^{(m)}-\varepsilon_{0 k}^{(m)}\right|=2 \varepsilon_{\mathrm{T} k}^{(m)},\left|\varepsilon_{k}^{(m)}\right|<\left|\varepsilon_{0 k}^{(m)}\right| \text { при }|z|=H_{m}\left(x_{1}, x_{2}\right),
$$

то на внешних поверхностях $m$-ного и $(m+M)$-ного слоев $\left(|z|=H_{m}\right)$ в волокнах $k$-того семейства с учетом идеального эффекта Баушингера [7] достигается вторичная пластичность (противоположная по знаку первичной пластичности). 
Если в точке $\left(x_{1}, x_{2}\right)$ связующее разгружается (в отличие от волокон, одна часть семейств которых в данный момент времени в рассматриваемой точке может разгружаться, а другая часть - догружаться, материал связующего во всех слоях в точках с координатами $|z| \leq H$ ( $x_{1}, x_{2}$ фиксированы) одновременно либо разгружается, либо догружается, что является следствием выражения для интенсивности деформаций в связующем (17)), то компоненты напряжения в нем определяются равенствами [13]

$$
\begin{aligned}
& \sigma_{\mathrm{c} i i}^{(m)}=-z E^{(m)} a_{1}^{(m)}\left(w_{, i i}+v^{(m)} w_{, j}\right), \quad \sigma_{\mathrm{c} i j}^{(m)}=-z E^{(m)} a_{2}^{(m)} w_{i j} \quad\left(H_{m-1}<|z| \leq h_{0}^{(m)}\right), \\
& \sigma_{\mathrm{c} i i}^{(m)}=\sigma_{0 i i}^{(m)}-2 z E^{(m)}\left(2 w_{, i i}+w_{, j j}\right) / 3+2 z E^{(m)}\left(2 w_{0},_{i i}+w_{0},{ }_{j j}\right) / 3, \\
& \sigma_{\mathrm{c} i j}^{(m)}=\sigma_{0 i j}^{(m)}-2 z E^{(m)}\left(w_{, i j}-w_{0},_{i j}\right) / 3, \quad j=3-i, \quad i=1,2 \quad\left(h_{0}^{(m)}<|z| \leq H_{m}\right),
\end{aligned}
$$

где согласно (16), (23)

$$
\begin{aligned}
& \sigma_{0 i i}^{(m)}=-2\left[z E_{*}^{(m)}+\operatorname{sign}(z)\left(\sigma_{\mathrm{T}}^{(m)}-E_{*}^{(m)} \varepsilon_{*}^{(m)}\right) / e_{u}^{0}\right]\left(2 w_{0},_{i i}+w_{0}, j j\right) / 3, \\
& \sigma_{0 i j}^{(m)}=-2\left[z E_{*}^{(m)}+\operatorname{sign}(z)\left(\sigma_{\mathrm{T}}^{(m)}-E_{*}^{(m)} \varepsilon_{*}^{(m)}\right) / e_{u}^{0}\right] w_{0},{ }_{i j} / 3, \quad j=3-i, \quad i=1,2 \quad\left(h_{0}^{(m)}<z \leq H_{m}\right) \text {, }
\end{aligned}
$$

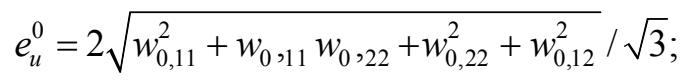

$h_{0}^{(m)}, w_{0},_{i j}-$ значения функций $h^{(m)}, w_{i j}$ соответственно (см. (21)), определенных в момент времени $t=t_{*}\left(x_{1}, x_{2}\right)$ начала разгрузки связующего в точке $\left(x_{1}, x_{2}\right)$ во всех слоях, который характеризуется соотношениями

$$
e_{u},\left(x_{1}, x_{2}, t_{*}\right)=0, e_{u}, t t\left(x_{1}, x_{2}, t_{*}\right)<0,
$$

где следует учесть выражение (14).

В соотношениях (31) в точках $h_{0}^{(m)}<|z| \leq H_{m}$ коэффициент Пуассона связующего $v^{(m)}=0,5$, что соответствует отсутствию дилатации. При задании истинного значения $v^{(m)}$ в этих точках получим разрыв напряжений $\sigma_{\text {сij }}^{(m)}$ в момент $t_{*}$ начала разгрузки в пластической зоне связующего $m$-ного слоя, в которой объемное сжатие до момента $t_{*}$ не учитывалось.

Если в процессе разгрузки связующего в точке $\left(x_{1}, x_{2}\right)$ выполняются соотношения

$$
e_{u \Delta}\left(x_{1}, x_{2}, t\right)=0, e_{u}, t\left(x_{1}, x_{2}, t\right)>0 \quad\left(t>t_{*}\right),
$$

где

$$
e_{u \Delta} \equiv \frac{2}{\sqrt{3}} \sqrt{\left(w_{, 11}-w_{0},_{11}\right)^{2}+\left(w_{, 11}-w_{0}, r_{11}\right)\left(w_{,_{22}}-w_{0,22}\right)+\left(w_{, 22}-w_{0}, r_{22}\right)^{2}+\left(w_{, 12}-w_{0},_{12}\right)^{2}},
$$

то в связующем вновь будет развиваться первичная пластичность и от равенств (31), (32) следует вернуться к (22), (23). Если же в процессе разгрузки связующего хотя бы для одного $m$-го слоя выполняется равенство

$$
e_{u \Delta}\left(x_{1}, x_{2}, t\right)=2 \varepsilon_{*}^{(m)} / H_{m}\left(x_{1}, x_{2}\right) \quad(m=0,1, \ldots, M),
$$

то на внешних поверхностях $m$-ного и $(m+M)$-ного слоев $\left(|z|=H_{m}\right)$ в связующем с учетом идеального эффекта Баушингера достигается вторичная пластичность [13].

Можно выписать связь между напряжениями $\sigma_{k}^{(m)}, \sigma_{\mathrm{c} i j}^{(m)}$ в фазах композиции и прогибом пластины $w$ при развитии вторичной пластичности и последующей «вторичной» разгрузке субструктурных элементов, а также и при последующих циклах знакопеременной пластичности. Однако это выходит за рамки настоящего исследования, тем более что с увеличением числа циклов знакопеременной пластичности такая связь становится все более громоздкой.

Подставим выражения (26), (31) в равенства (3), а последние в первое соотношение (2). Тогда после вычисления интегралов получим представление для моментов при разгрузке всех или части субструктурных элементов конструкции, совпадающее с (24), где коэффициенты имеют вид 


$$
\begin{aligned}
& C_{i i i i}=\sum_{m=0}^{M}\left\{C_{i i i i}^{0}\left(x_{1}, x_{2} ; w\right)+\sum_{\alpha} \omega_{\alpha}^{(m)}\left(l_{\alpha i}^{(m)}\right)^{4}\left[E_{\alpha}^{(m)}\left(\left(h_{\alpha}^{(m)}\right)^{3}-H_{m-1}^{3}\right)+E_{\mathrm{\tau} \alpha}^{(m)}\left(H_{m}^{3}-\left(h_{\alpha}^{(m)}\right)^{3}\right)\right]+\right. \\
& \left.+\sum_{\beta} \omega_{\beta}^{(m)}\left(l_{\beta i}^{(m)}\right)^{4} E_{\beta}^{(m)}\left(H_{m}^{3}-H_{m-1}^{3}\right)\right\}, C_{i i j j}=\sum_{m=0}^{M}\left\{C_{i i j j}^{0}\left(x_{1}, x_{2} ; w\right)+\sum_{\alpha} \omega_{\alpha}^{(m)}\left(l_{\alpha i}^{(m)}\right)^{2}\left(l_{\alpha j}^{(m)}\right)^{2} \times\right. \\
& \left.\times\left[E_{\alpha}^{(m)}\left(\left(h_{\alpha}^{(m)}\right)^{3}-H_{m-1}^{3}\right)+E_{\mathrm{\tau} \alpha}^{(m)}\left(H_{m}^{3}-\left(h_{\alpha}^{(m)}\right)^{3}\right)\right]+\sum_{\beta} \omega_{\beta}^{(m)}\left(l_{\beta i}^{(m)}\right)^{2}\left(l_{\beta j}^{(m)}\right)^{2} E_{\beta}^{(m)}\left(H_{m}^{3}-H_{m-1}^{3}\right)\right\}, \\
& C_{i i i j}=\sum_{m=0}^{M}\left\{\sum_{\alpha} \omega_{\alpha}^{(m)}\left(l_{\alpha i}^{(m)}\right)^{3} l_{\alpha j}^{(m)}\left[E_{\alpha}^{(m)}\left(\left(h_{\alpha}^{(m)}\right)^{3}-H_{m-1}^{3}\right)+E_{\mathrm{T \alpha}}^{(m)}\left(H_{m}^{3}-\left(h_{\alpha}^{(m)}\right)^{3}\right)\right]+\right. \\
& \left.+\sum_{\beta} \omega_{\beta}^{(m)}\left(l_{\beta i}^{(m)}\right)^{3} l_{\beta j}^{(m)} E_{\beta}^{(m)}\left(H_{m}^{3}-H_{m-1}^{3}\right)\right\}, C_{i j i j}=\sum_{m=0}^{M}\left\{C_{i j i j}^{0}\left(x_{1}, x_{2} ; w\right)+\sum_{\alpha} \omega_{\alpha}^{(m)}\left(l_{\alpha i}^{(m)}\right)^{2}\left(l_{\alpha j}^{(m)}\right)^{2} \times\right. \\
& \left.\times\left[E_{\alpha}^{(m)}\left(\left(h_{\alpha}^{(m)}\right)^{3}-H_{m-1}^{3}\right)+E_{\mathrm{\tau} \alpha}^{(m)}\left(H_{m}^{3}-\left(h_{\alpha}^{(m)}\right)^{3}\right)\right]+\sum_{\beta} \omega_{\beta}^{(m)}\left(l_{\beta i}^{(m)}\right)^{2}\left(l_{\beta j}^{(m)}\right)^{2} E_{\beta}^{(m)}\left(H_{m}^{3}-H_{m-1}^{3}\right)\right\} \\
& (j=3-i, i=1,2), D_{i j}=\sum_{m=0}^{M}\left\{3 \sum_{\alpha} \omega_{\alpha}^{(m)} l_{\alpha i}^{(m)} l_{\alpha j}^{(m)} \operatorname{sign}\left(e_{\alpha}^{(m)}\right)\left(H_{m}^{2}-\left(h_{\alpha}^{(m)}\right)^{2}\right)\left(\sigma_{\mathrm{\tau} \alpha}^{(m)}-E_{\mathrm{T \alpha}}^{(m)} \varepsilon_{\mathrm{\tau} \alpha}^{(m)}\right)+\right. \\
& +\sum_{\beta} \omega_{\beta}^{(m)} l_{\beta i}^{(m)} l_{\beta j}^{(m)}\left[e_{0 \beta}^{(m)}\left(E_{\mathrm{\tau} \beta}^{(m)}-E_{\beta}^{(m)}\right)\left(H_{m}^{3}-\left(h_{0 \beta}^{(m)}\right)^{3}\right)+3 \operatorname{sign}\left(e_{0 \beta}^{(m)}\right)\left(\sigma_{\mathrm{\tau} \beta}^{(m)}-E_{\mathrm{\tau} \beta}^{(m)} \varepsilon_{\mathrm{\tau} \beta}^{(m)}\right) \times\right. \\
& \left.\left.\times\left(H_{m}^{2}-\left(h_{0 \beta}^{(m)}\right)^{2}\right)\right]\right\}+D_{i j}^{0}\left(x_{1}, x_{2} ; w\right)(i, j=1,2), H_{-1} \equiv 0 .
\end{aligned}
$$

Здесь индекс $\alpha$ пробегает значения от 1 до $N^{(m)}$ только для тех номеров семейств волокон $m$-го слоя, в которых продолжается догрузка; индекс $\beta$ пробегает значения от 1 до $N^{(m)}$ только для тех номеров семейств волокон $m$-го слоя, в которых имеет место разгрузка $\left(1 \leq \alpha, \beta \leq N^{(m)}, \alpha \neq \beta\right)$. При догрузке связующего коэффициенты $C_{i j s l}^{0}, D_{i j}^{0}$ в (37) имеют выражения

$$
\begin{aligned}
& C_{i i i i}^{0}=\sum_{m=0}^{M}\left\{a ^ { ( m ) } \left[a_{1}^{(m)} E^{(m)}\left(\left(h^{(m)}\right)^{3}-H_{m-1}^{3}\right)+4\left[\frac{1}{3} E_{*}^{(m)}\left(H_{m}^{3}-\left(h^{(m)}\right)^{3}\right)+\left(\sigma_{\mathrm{T}}^{(m)}-E_{*}^{(m)} \varepsilon_{*}^{(m)}\right) \times\right.\right.\right. \\
& \left.\left.\times \frac{1}{e_{u}}\left(H_{m}^{2}-\left(h^{(m)}\right)^{2}\right)\right]\right\}, C_{i i j j}^{0}=\sum_{m=0}^{M}\left\{a ^ { ( m ) } \left[a_{1}^{(m)} E^{(m)} v^{(m)}\left(\left(h^{(m)}\right)^{3}-H_{m-1}^{3}\right)+2\left[\frac{1}{3} E_{*}^{(m)}\left(H_{m}^{3}-\left(h^{(m)}\right)^{3}\right)+\right.\right.\right. \\
& \left.\left.+\frac{1}{e_{u}}\left(\sigma_{\mathrm{T}}^{(m)}-E_{*}^{(m)} \varepsilon_{*}^{(m)}\right)\left(H_{m}^{2}-\left(h^{(m)}\right)^{2}\right)\right]\right\}, C_{i j i j}^{0}=\sum_{m=0}^{M} a^{(m)}\left[\frac{1}{2} a_{2}^{(m)} E^{(m)}\left(\left(h^{(m)}\right)^{3}-H_{m-1}^{3}\right)+\right. \\
& \left.+\frac{1}{3} E_{*}^{(m)}\left(H_{m}^{3}-\left(h^{(m)}\right)^{3}\right)+\frac{1}{e_{u}}\left(\sigma_{\mathrm{T}}^{(m)}-E_{*}^{(m)} \varepsilon_{*}^{(m)}\right)\left(H_{m}^{2}-\left(h^{(m)}\right)^{2}\right)\right], C_{i i i j}^{0}=0 \quad(j=3-i, i=1,2), \\
& D_{i j}^{0}=0(i, j=1,2), H_{-1} \equiv 0,
\end{aligned}
$$

совпадающие с соответствующими слагаемыми в (25); при разгрузке связующего вместо (38) получаем

$$
\begin{aligned}
& C_{i i i i}^{0}=\sum_{m=0}^{M} a^{(m)} E^{(m)}\left[a_{1}^{(m)}\left(\left(h_{0}^{(m)}\right)^{3}-H_{m-1}^{3}\right)+\frac{4}{3}\left(H_{m}^{3}-\left(h_{0}^{(m)}\right)^{3}\right)\right], C_{i i j j}^{0}=\sum_{m=0}^{M} a^{(m)} E^{(m)} \times \\
& \times\left[a_{1}^{(m)} v^{(m)}\left(\left(h_{0}^{(m)}\right)^{3}-H_{m-1}^{3}\right)+\frac{2}{3}\left(H_{m}^{3}-\left(h_{0}^{(m)}\right)^{3}\right)\right], C_{i j i j}^{0}=\sum_{m=0}^{M} a^{(m)} E^{(m)}\left[0,5 a_{2}^{(m)}\left(\left(h_{0}^{(m)}\right)^{3}-H_{m-1}^{3}\right)+\right. \\
& \left.+\frac{1}{3}\left(H_{m}^{3}-\left(h_{0}^{(m)}\right)^{3}\right)\right], C_{i i i j}^{0}=0, D_{i i}^{0}=-2\left(2 w_{0},_{i i}+w_{0}, j j\right) \sum_{m=0}^{M} a^{(m)}\left[\frac{1}{3}\left(E_{*}^{(m)}-E^{(m)}\right)\left(H_{m}^{3}-\left(h_{0}^{(m)}\right)^{3}\right)+\right. \\
& \left.+\frac{1}{e_{u}^{0}}\left(\sigma_{\mathrm{T}}^{(m)}-E_{*}^{(m)} \varepsilon_{*}^{(m)}\right)\left(H_{m}^{2}-\left(h_{0}^{(m)}\right)^{2}\right)\right], D_{i j}^{0}=-2 w_{0} r_{i j} \sum_{m=0}^{M} a^{(m)}\left[\frac{1}{3}\left(E_{*}^{(m)}-E^{(m)}\right)\left(H_{m}^{3}-\left(h_{0}^{(m)}\right)^{3}\right)+\right.
\end{aligned}
$$


$\left.+\frac{1}{e_{u}^{0}}\left(\sigma_{\mathrm{T}}^{(m)}-E_{*}^{(m)} \varepsilon_{*}^{(m)}\right)\left(H_{m}^{2}-\left(h_{0}^{(m)}\right)^{2}\right)\right] \quad(j=3-i, \quad i=1,2), \quad H_{-1} \equiv 0$,

где функция $e_{u}^{0}$ определена в (32). Если не учитывается сжимаемость в упругих зонах связующего $\left(v^{(m)}=0,5\right)$, то коэффициенты $C_{i j s}^{0}, D_{i j}^{0}$ в (39) редуцируются к

$$
\begin{aligned}
& C_{i i i i}^{0}=\frac{4}{3} \sum_{m=0}^{M} a^{(m)} E^{(m)}\left(H_{m}^{3}-H_{m-1}^{3}\right), \quad C_{i i j j}^{0}=\frac{2}{3} \sum_{m=0}^{M} a^{(m)} E^{(m)}\left(H_{m}^{3}-H_{m-1}^{3}\right), \\
& C_{i j i j}^{0}=\frac{1}{3} \sum_{m=0}^{M} a^{(m)} E^{(m)}\left(H_{m}^{3}-H_{m-1}^{3}\right), C_{i i i j}^{0}=0 \quad(j=3-i, \quad i=1,2), H_{-1} \equiv 0, v^{(m)}=0,5 .
\end{aligned}
$$

Таким образом, до появления вторичной пластичности хотя бы в одной из фаз композиции моменты в пластине определяются равенствами (24) с учетом соотношений (25) или (37)—(40), последние же являются обобщением равенств (25).

В случае пространственного армирования тонких слоев пластины непрерывными волокнами постоянного поперечного сечения между параметрами армирования существует связь, приближенно определяемая равенством [14]:

$$
\left[\omega_{k}^{(m)}\left(H_{m}-H_{m-1}\right) \cos \psi_{k}^{(m)}\right]_{, 1}+\left[\omega_{k}^{(m)}\left(H_{m}-H_{m-1}\right) \sin \psi_{k}^{(m)}\right]_{, 2}=0 \quad\left(H_{-1} \equiv 0\right),
$$

которое при плоском армировании редуцируется к точному соотношению

$$
\left(\omega_{k}^{(m)} \cos \psi_{k}^{(m)}\right)_{, 1}+\left(\omega_{k}^{(m)} \sin \psi_{k}^{(m)}\right)_{, 2}=0,1 \leq k \leq N^{(m)}, 0 \leq m \leq M .
$$

Пусть область $G$, занимаемая пластиной в плане, ограничена контуром $\Gamma$, тогда на одной части этого контура (обозначим ее $\Gamma_{p}$ ) могут быть заданы граничные условия по изгибающему моменту [8]

$$
M_{11} n_{1}^{2}+M_{22} n_{2}^{2}+2 M_{12} n_{1} n_{2}=M_{n}, \quad n_{1}=\cos \gamma, n_{2}=\sin \gamma, \quad\left(x_{1}, x_{2}\right) \in \Gamma_{p}
$$

и приведенной поперечной силе Кирхгофа

$$
\begin{aligned}
& F_{1} n_{1}+F_{2} n_{2}+\partial_{\tau}\left(M_{n \tau}\right)=F_{n z}, \quad M_{n \tau} \equiv\left(M_{22}-M_{11}\right) n_{1} n_{2}+ \\
& +M_{12}\left(n_{1}^{2}-n_{2}^{2}\right), \quad \partial_{\tau}\left(M_{n \tau}\right)=-n_{2} M_{n \tau}, 1+n_{1} M_{n \tau}, \quad\left(x_{1}, x_{2}\right) \in \Gamma_{p},
\end{aligned}
$$

а на другой части (обозначим ее $\Gamma_{u}$ ) 一 кинематические граничные условия

$$
w\left(\Gamma_{u}\right)=w_{n}, \quad w_{, 1} n_{1}+w_{2} n_{2}=\theta_{n}, \quad\left(x_{1}, x_{2}\right) \in \Gamma_{u},
$$

где $M_{n}, F_{n z}$ - изгибающий момент и приведенная поперечная сила Кирхгофа, заданные на $\Gamma_{p} ; w_{n}, \theta_{n}$ - прогиб на $\Gamma_{u}$ и производная от прогиба по направлению внешней нормали к контуру, задаваемой углом $\gamma ; \partial_{\tau}$ - производная вдоль контура. (На контуре Г могут быть заданы и смешанные из (43)- (45) граничные условия, например условия свободного опирания.)

На той части контура Г (обозначим ее $\Gamma_{k}^{(m)}$ ), на которой волокна $k$-го семейства $m$-го слоя входят в область $G$, необходимо задать краевые условия для интенсивностей армирования [14]:

$$
\omega_{k}^{(m)}\left(\Gamma_{k}^{(m)}\right)=\omega_{0 k}^{(m)}, k=1,2, \ldots, N^{(m)}, \quad m=0,1, \ldots, M,
$$

где $\omega_{0 k}^{(m)}$ - заданные на $\Gamma_{k}^{(m)}$ функции.

В начальный момент времени $t=t_{0}$ должны выполняться начальные условия для прогиба и его скорости

$$
w\left(x_{1}, x_{2}, t_{0}\right)=w^{0}\left(x_{1}, x_{2}\right), w_{t}\left(x_{1}, x_{2}, t_{0}\right)=v^{0}\left(x_{1}, x_{2}\right), \quad\left(x_{1}, x_{2}\right) \in G,
$$

где $w^{0}, v^{0}-$ заданные функции.

Решение задачи о динамическом изгибе армированной слоистой пластины должно удовлетворять прочностным ограничениям $[7,8,11]$

$$
\begin{aligned}
& \sigma_{u}^{(m)}\left(x_{1}, x_{2}, H_{m}, t\right) \leq \sigma_{\mathrm{c}}^{(m)}, \quad\left|\sigma_{k}^{(m)}\left(x_{1}, x_{2}, H_{m}, t\right)\right| \leq \sigma_{k}^{*(m)}=\min \left(\sigma_{k}^{-(m)}, \sigma_{k}^{+(m)}\right), \\
& \sigma_{\mathrm{c}}^{(m)}>0, \quad \sigma_{k}^{ \pm(m)}>0, \quad 1 \leq k \leq N^{(m)}, 0 \leq m \leq M,
\end{aligned}
$$

где $\sigma_{\mathrm{c}}^{(m)}$ - предел прочности связующего $m$-го слоя, равный пределу текучести $\sigma_{\mathrm{T}}^{(m)}$ при упругом изгибе или временному сопротивлению $\sigma_{\mathrm{B}}^{(m)}$ при упругопластическом изгибе; $\sigma_{k}^{ \pm(m)}$ пределы прочности волокон $k$-го семейства $m$-го слоя при сжатии (-) и растяжении (+) (в сжа- 
той зоне может возникнуть некоторая форма неустойчивости волокон, поэтому в общем случае $\left.\sigma_{k}^{-(m)} \neq \sigma_{k}^{+(m)}\right)$.

Структура армирования слоев должна удовлетворять физическим неравенствам

$$
0 \leq \omega_{k}^{(m)} \quad\left(k=1,2, \ldots, N^{(m)}\right), \quad \sum_{k} \omega_{k}^{(m)} \leq \omega_{*}^{(m)} \leq 1 \quad(m=0,1,2, \ldots, M),
$$

где $\omega_{*}^{(m)}=$ const - предельно допустимая суммарная интенсивность армирования $m$-го слоя.

Для получения разрешающего уравнения упругопластического динамического изгиба армированных слоистых пластин и соответствующих ему граничных условий в прогибах необходимо соотношения (24) подставить в уравнения движения (1) и граничные условия (43), (44) и исключить из рассмотрения поперечные силы $F_{i}$. Тогда уравнение движения примет вид

$$
R\left(x_{1}, x_{2}\right) w_{, t}=p\left(x_{1}, x_{2}, t\right)-\frac{2}{3} \sum_{i=1}^{2} \sum_{j=1}^{2}\left(\sum_{s=1}^{2} \sum_{l=1}^{2} C_{i j s l}\left(x_{1}, x_{2} ; w\right) w_{s l}-D_{i j}\left(x_{1}, x_{2} ; w\right)\right), i j,
$$

а граничные условия по силовым факторам преобразуются к виду: по изгибающему моменту

$$
\begin{aligned}
& -\left(C_{1111} \cos ^{2} \gamma+C_{1122} \sin ^{2} \gamma+C_{1112} \sin 2 \gamma\right) w_{, 11}-\left(C_{2211} \cos ^{2} \gamma+C_{2222} \sin ^{2} \gamma+C_{2212} \sin 2 \gamma\right) w_{, 22} \\
& -2\left(C_{1211} \cos ^{2} \gamma+C_{1222} \sin ^{2} \gamma+C_{1212} \sin 2 \gamma\right) w_{, 12}+D_{11} \cos ^{2} \gamma+D_{22} \sin ^{2} \gamma+D_{12} \sin 2 \gamma=3 M_{n} / 2 ;
\end{aligned}
$$

по приведенной силе Кирхгофа

$$
\begin{aligned}
& -\sum_{i=1}^{2} \sum_{j=1}^{2}\left(\sum_{s=1}^{2} \sum_{l=1}^{2} C_{i j s l} w,_{s l}-D_{i j}\right),{ }_{j} n_{i}-\frac{1}{2} \partial_{\tau}\left\{\left[\left(C_{1122}-C_{1111}\right) \sin 2 \gamma+2 C_{1112} \cos 2 \gamma\right] w_{11}+\right. \\
& +\left[\left(C_{2222}-C_{2211}\right) \sin 2 \gamma+2 C_{2212} \cos 2 \gamma\right] w_{, 22}+2\left[\left(C_{1222}-C_{1211}\right) \sin 2 \gamma+2 C_{1212} \cos 2 \gamma\right] w_{12}- \\
& \left.-\left(D_{22}-D_{11}\right) \sin 2 \gamma-2 D_{12} \cos 2 \gamma\right\}=3 F_{n z} / 2, \quad\left(x_{1}, x_{2}\right) \in \Gamma_{p},
\end{aligned}
$$

где функции $M_{n}, F_{n z}$ могут зависеть от времени $t$ и координаты, заданной вдоль контура $\Gamma_{p}$, а коэффициенты $C_{i j m l}, D_{i j}$ имеют выражения (25) или (37)—(40) и нелинейно зависят от $w_{i j}, H_{m}$ и параметров армирования $\psi_{k}^{(m)}, \omega_{k}^{(m)}$. При линейно-упругом поперечном изгибе в (25), (37)-(39) следует принять $h_{k}^{(m)}=h^{(m)}=h_{0 k}^{(m)}=h_{0}^{(m)}=H_{m}$, тогда получим линейные относительно прогиба уравнение движения (50), граничные условия (51), (52) и выражения для моментов (24).

Если толщины слоев (или, что то же самое, аппликаты границ между слоями $z=H_{m}$ ) и траектории армирования (т.е. углы $\psi_{k}^{(m)}$ ) каждого слоя заданы, то краевая задача (41), (46) или (42), (46) определяет интенсивности армирования $\omega_{k}^{(m)} m$-го слоя волокнами $k$-го семейства. (Краевые задачи для линейных уравнений в частных производных первого порядка (41), (42) хорошо изучены [15], поэтому не будем останавливаться на этом вопросе более подробно; отметим лишь, что уравнения (41), (42) имеют действительные характеристики, совпадающие с траекториями армирования $k$-го семейства $m$-го слоя.)

Если функции $\psi_{k}^{(m)}, \omega_{k}^{(m)}, H_{m}$ известны (заданы) и удовлетворяют физическим ограничениям (49), то уравнение движения (50) замкнуто относительно прогиба $w$. Этому квазилинейному (в случае упругопластического изгиба) или линейному (в случае линейно-упругого изгиба) параболическому дифференциальному уравнению в частных производных (содержащему производные второго порядка по времени $t$ и четвертого порядка по пространственным переменным $x_{i}$ ) соответствуют нелинейные или линейные граничные (45), (51), (52) и начальные (47) условия. Решение сформулированной начально-краевой задачи упругопластического динамического изгиба слоисто-волокнистых пластин должно удовлетворять прочностным ограничениям (48).

Численный метод интегрирования начально-краевой задачи упругопластической динамики композитных пластин. Для приближенного интегрирования уравнения (50) по времени $t$ используем обобщенные методы Рунге-Кутта $[16,17]$. С этой целью уравнение (50) разделим на $R$ и перепишем в виде системы

$$
w_{, t}(\mathbf{x}, t)=v(\mathbf{x}, t), \quad v_{t}(\mathbf{x}, t)=q(\mathbf{x}, t)-L(\mathbf{x} ; w),
$$




$$
L(\mathbf{x} ; w)=\frac{2}{3 R(\mathbf{x})} \sum_{i=1}^{2} \sum_{j=1}^{2}\left(\sum_{s=1}^{2} \sum_{l=1}^{2} C_{i j s l}(\mathbf{x} ; w) w_{s_{s l}}(\mathbf{x}, t)-D_{i j}(\mathbf{x} ; w)\right),,_{i j}, q(\mathbf{x}, t)=\frac{p(\mathbf{x}, t)}{R(\mathbf{x})}, \quad \mathbf{x}=\left\{\begin{array}{l}
x_{1} \\
x_{2}
\end{array}\right\} .
$$

Численно систему (53) проинтегрируем с помощью двустадийного обобщенного метода Лобатто IIIA - метода трапеций (наименования методов будем использовать в соответствии с принятой в [18] терминологией), имеющего второй порядка точности по $\tau$, где $\tau-$ шаг по времени $t$. Согласно основной идее обобщения методов Рунге-Кутта $[16,17]$ этот метод применительно к системе (53) реализуется так:

$$
\begin{aligned}
& w^{n+1}=w^{n}+\tau\left(V_{1}+V_{2}\right) / 2, v^{n+1}=v^{n}+\tau\left(q^{n}-L\left(\mathbf{x} ; W_{1}\right)+q^{n+1}-L\left(\mathbf{x} ; W_{2}\right)\right) / 2, \\
& W_{1}=w^{n}+0 \cdot \tau\left(V_{1}+V_{2}\right), W_{2}=w^{n}+\tau\left(V_{1}+V_{2}\right) / 2, \\
& V_{1}=v^{n}+0 \cdot \tau\left(q^{n}-L\left(\mathbf{x} ; W_{1}\right)+q^{n+1}-L\left(\mathbf{x} ; W_{2}\right)\right), V_{2}=v^{n}+\tau\left(q^{n}-L\left(\mathbf{x} ; W_{1}\right)+q^{n+1}-L\left(\mathbf{x} ; W_{2}\right)\right) / 2,
\end{aligned}
$$

откуда после несложных преобразований получим

$$
\begin{aligned}
& w^{n+1}=W_{2}(\mathbf{x}), \quad w^{n}=W_{1}(\mathbf{x}), \quad v^{n+1}=V_{2}(\mathbf{x}), \quad v^{n}=V_{1}(\mathbf{x}), \\
& v^{n+1}=2\left(w^{n+1}-w^{n}\right) / \tau-v^{n}, \quad v^{n+1}=v^{n}+\tau\left(q^{n}-L\left(\mathbf{x} ; w^{n}\right)+q^{n+1}-L\left(\mathbf{x} ; w^{n+1}\right)\right) / 2 ; \\
& \tau^{2} D\left(\mathbf{x} ; w^{n+1}\right)+4 R(\mathbf{x}) w^{n+1}=\tau^{2}\left(p^{n}+p^{n+1}-D\left(\mathbf{x} ; w^{n}\right)\right)+4 R(\mathbf{x})\left(\tau v^{n}+w^{n}\right),
\end{aligned}
$$

где

$$
\begin{gathered}
D\left(\mathbf{x} ; w^{n}\right)=R(\mathbf{x}) L\left(\mathbf{x} ; w^{n}\right)=\frac{2}{3} \sum_{i=1}^{2} \sum_{j=1}^{2}\left(\sum_{s=1}^{2} \sum_{l=1}^{2} C_{i j s l}\left(\mathbf{x} ; w^{n}\right) w_{s l}^{n}(\mathbf{x})-D_{i j}\left(\mathbf{x} ; w^{n}\right)\right),{ }_{i j}, q^{n}(\mathbf{x})=q\left(\mathbf{x}, t_{n}\right), \\
p^{n}(\mathbf{x})=p\left(\mathbf{x}, t_{n}\right), w^{n}(\mathbf{x})=w\left(\mathbf{x}, t_{n}\right), v^{n}(\mathbf{x})=v\left(\mathbf{x}, t_{n}\right), t_{n+1}=t_{n}+\tau, n=0,1,2 \ldots \quad\left(t_{0}=0\right) ;
\end{gathered}
$$

шаг по времени $\tau>0$ может быть переменным $\left(\tau=\tau_{n}\right) ; W_{1}, W_{2}, V_{1}, V_{2}-$ вспомогательные функции.

Если решение на $n$-ом «слое» по времени известно (известны $w^{n}, v^{n}$ ), то уравнение (56) задает решение на следующем $(n+1)$-ом «слое». Определенным недостатком уравнения (56) является то, что для вычисления его правой части необходимо применить дифференциальный оператор четвертого порядка $D(\mathbf{x} ; \bullet)$ к известной функции $w^{n}$ (см. (56), (57)). Чтобы избежать этого дифференцирования, введем в рассмотрение функции

$$
P_{n}(\mathbf{x})=\tau^{2} D\left(\mathbf{x} ; w^{n}\right)+4 R(\mathbf{x}) w^{n}, \quad n=0,1,2 \ldots .
$$

Тогда разрешающее уравнение (56) примет вид

$$
\tau^{2} D\left(\mathbf{x} ; w^{n+1}\right)+4 R(\mathbf{x}) w^{n+1}=P_{n+1}(\mathbf{x}),
$$

где правая часть известна и определяется по рекуррентной формуле

$$
P_{n+1}(\mathbf{x})=-P_{n}(\mathbf{x})+4 R(\mathbf{x})\left(\tau v^{n}+2 w^{n}\right)+\tau^{2}\left(p^{n}+p^{n+1}\right),
$$

вытекающей из сравнения (58) и правой части (56). В момент времени $t=t_{0}$ в силу начальных условий (47) из (58) получим известную функцию

$$
P_{0}(\mathbf{x})=\tau^{2} D\left(\mathbf{x} ; w^{0}(\mathbf{x})\right)+4 R(\mathbf{x}) w^{0}(\mathbf{x}) .
$$

В частности, при нулевых начальных условиях

$$
P_{0}(\mathbf{x})=0 \quad\left(v^{0}(\mathbf{x})=0, \quad w^{0}(\mathbf{x})=0\right),
$$

а из (60), (62) следует

$$
P_{1}(\mathbf{x})=\tau^{2}\left(p^{0}(\mathbf{x})+p^{1}(\mathbf{x})\right) .
$$

Таким образом, для определения прогиба на $(n+1)$-ом «слое» по времени необходимо проинтегрировать уравнение (59) с известной правой частью (60)-(63) при граничных условиях, которые полностью соответствуют граничным условиям исходной начально-краевой задачи. (В частности, в граничных условиях (45), (51), (52) необходимо заменить $w$ на $w^{n+1}(\mathbf{x})$.)

Уравнение (59) формально совпадает с уравнением статического упругопластического изгиба слоисто-волокнистой пластины на упругом основании. Для интегрирования граничной задачи, соответствующей уравнению (59), можно использовать известные методы статики. 
Основная трудность, возникающая при решении граничной задачи для уравнения (59), заключается в ее существенной нелинейности при наличии пластических зон в фазах композиции. Для линеаризации задачи упругопластического изгиба можно использовать следующий итерационный процесс, качественно аналогичный методу переменных параметров упругости [7]. Пусть $w_{(r)}^{n+1}(\mathbf{x})-$ известное $r$-е приближение разыскиваемой функции $w^{n+1}$. Тогда в (51), (52), (57) по формулам (25), (37)—(40) с учетом (8), (16), (21) определим $r$-ые приближения $C_{i j s l(r)}, D_{i j(r)}$ коэффициентов $C_{i j s l}, D_{i j}$, после чего вместо (59) получим линейное уравнение

$$
\tau^{2} D_{(r)}\left(\mathbf{x} ; w_{(r+1)}^{n+1}(\mathbf{x})\right)+4 R(\mathbf{x}) w_{(r+1)}^{n+1}(\mathbf{x})=P_{n+1}(\mathbf{x})
$$

и соответствующие ему линейные граничные условия (45), (51), (52), в которых (а также и в (64) с учетом (57)) следует $w^{n+1}$ заменить на $w_{(r+1)}^{n+1}$ и $C_{i j s l}, D_{i j}$ - на приближения $C_{i j s l(r)}, D_{i j(r)}$.

В качестве начального приближения для $w^{n+1}$ можно выбрать функцию

$$
w_{(0)}^{n+1}(\mathbf{x})=w^{n}(\mathbf{x})+\tau v^{n}(\mathbf{x}),
$$

получающуюся по формуле Тейлора в предположении, что на предыдущем $n$-ом «слое» по времени решение задачи уже известно. Авторам пока не удалось доказать сходимость такого итерационного метода, но многочисленные расчеты показывают, что он всегда сходится в смысле ограниченности нормы приближения решения и стремления к нулю нормы разности двух последовательных приближений решения при увеличении количества итераций.

При решении граничной задачи для уравнения (59) (или (64)) следует учитывать, что диаграммы деформирования фазовых материалов предполагаются кусочно-линейными. Поэтому в точках излома этих диаграмм функции $\sigma_{u}^{(m)}\left(\varepsilon_{u}\right), \sigma_{k}^{(m)}\left(\varepsilon_{k}^{(m)}\right)\left(1 \leq k \leq N^{(m)}, 0 \leq m \leq M\right)$ непрерывны, но их производные испытывают разрыв. Следовательно, частные производные от коэффициентов $C_{i j s l}, D_{i j}$ в (51), (52), (57) испытывают разрыв на линиях $\Gamma_{*}$, определяемых равенствами

$$
\Gamma_{*}: h_{k}^{(m)}=H_{m-1}, \quad h_{k}^{(m)}=H_{m}, h^{(m)}=H_{m-1}, \quad h^{(m)}=H_{m} \quad\left(1 \leq k \leq N^{(m)}, \quad 0 \leq m \leq M\right)
$$

(так как на этих линиях испытывают разрыв производные от функций $h_{k}^{(m)}, h^{(m)}$, см. (8), (21)), а значит, на линиях (66) необходимо ставить общеизвестные условия сопряжения решения [8]. Задача усложняется тем, что линии (66) изменяются при переходе от $n$-го «слоя» по времени $t_{n}$ к следующему $t_{n+1}$, а также при реализации итерационного процесса (64), (65). Чтобы избежать необходимости решения сопряженной задачи упругопластического изгиба при гладких структурах армирования слоев, для приближенного решения задачи целесообразно использовать энергетические методы, которые в ряде случаев за счет интегральных преобразований (интегрирование по частям, формула Грина и т.д.) позволяют избежать дифференцирования функций $C_{i j s l}, D_{i j}$. В частности, с этой целью можно использовать метод Бубнова-Галеркина.

Для интегрирования граничной задачи (45), (51), (52), (59) (или (45), (51), (52), (64)) методом Бубнова-Галеркина, как обычно, представим решение в виде суммы [19]

$$
w^{n+1}\left(x_{1}, x_{2}\right)=\sum_{i=1}^{K} W_{i}^{n+1} w_{i}\left(x_{1}, x_{2}\right) \quad(K \geq 1),
$$

где $w_{i}\left(x_{1}, x_{2}\right)$ - базисные функции, тождественно удовлетворяющие граничным условиям на всем контуре пластины; $W_{i}^{n+1}-$ коэффициенты, подлежащие определению. Для прямоугольных, эллиптических (односвязных и двусвязных), круглых и кольцевых пластин, жестко защемленных по всему контуру $Г$, базисные функции $w_{i}\left(x_{1}, x_{2}\right)$ приведены в [19]. В случае прямоугольной пластины вместо (67) можно использовать разложение вида

$$
w^{n+1}\left(x_{1}, x_{2}\right)=\sum_{i=1}^{K_{1}} \sum_{j=1}^{K_{2}} W_{i j}^{n+1} X_{1 i}\left(x_{1}\right) X_{2 j}\left(x_{2}\right) \quad\left(K_{1} \geq 1, K_{2} \geq 1\right) \text {, }
$$

где $X_{1 i}\left(x_{1}\right), X_{2 j}\left(x_{2}\right)$ - базисные функции, тождественно удовлетворяющие граничным условиям на противоположных сторонах прямоугольной пластины; $W_{i j}^{n+1}-$ коэффициенты, подлежащие определению. Если пластина жестко защемлена по всему контуру, то при любых структурах армирования в качестве $X_{1 i}\left(x_{1}\right), X_{2 j}\left(x_{2}\right)$ можно использовать известные балочные функции или другие базисные функции, приведенные в [19]. 
В случае шарнирного опирания прямоугольной пластины ( $\left.0 \leq x_{1} \leq c, 0 \leq x_{2} \leq b\right)$ имеем граничные условия

$$
\begin{gathered}
w\left(0, x_{2}\right)=w\left(c, x_{2}\right)=w\left(x_{1}, 0\right)=w\left(x_{1}, b\right)=0 ; \\
\left.M_{11}(w)\right|_{x_{1}=0, c}=\left.M_{22}(w)\right|_{x_{2}=0, b}=0,
\end{gathered}
$$

где $M_{i i}(w)$ определены в (24) с учетом (25), (37)—(40). В общем случае равенства (70) являются нелинейными граничными условиями по моменту. При линейно-упругом деформировании (в частности, при разгрузке всех фаз композиции) на кромках пластины моменты $M_{i i}$ суть линейные операторы от прогиба $w$.

Если структура армирования каждого слоя такова, что все $N^{(m)}=2 L^{(m)}$ ( $N^{(m)}-$ четное) семейств волокон $m$-го слоя можно разбить на пары так, что волокна семейств, образующих пару, изготовлены из одного материала и пересекают кромки прямоугольной пластины по симметричным направлениям $\left(\psi_{2 l-1}^{(m)}=-\psi_{2 l}^{(m)}\right)$ с одинаковой интенсивностью $\quad\left(\omega_{2 l-1}^{(m)}=\omega_{2 l}^{(m)}\right.$, $\left.l=1,2, \ldots, L^{(m)}, m=0,1, \ldots, M\right)$, то можно показать, что с учетом равенств (69) условия по моментам (70) эквивалентны кинематическим условиям

$$
w_{, 11}\left(0, x_{2}\right)=w_{, 11}\left(c, x_{2}\right)=w_{, 22}\left(x_{1}, 0\right)=w_{, 22}\left(x_{1}, b\right)=0 .
$$

Указанные типы структур существуют и достаточно разнообразны. Во-первых, к ним относятся прямолинейные структуры армирования ( $\psi_{k}^{(m)}=$ const $)$. Например, при $N^{(m)}=4$ : первое семейство волокон направлено вдоль $x_{1}\left(\psi_{1}^{(m)}=0\right)$, второе - вдоль $x_{2}\left(\psi_{2}^{(m)}=\pi / 2\right)$, третье и четвертое семейства - по направлениям $\psi_{3}^{(m)}=-\psi_{4}^{(m)}=$ const $\left(\omega_{3}^{(m)}=\omega_{4}^{(m)}=\right.$ const, а интенсивности армирования первого и второго семейств могут быть непостоянными, т.е. $\left.\omega_{1}^{(m)}=\omega_{1}^{(m)}\left(x_{2}\right), \omega_{2}^{(m)}=\omega_{2}^{(m)}\left(x_{1}\right)\right)$.

Во-вторых, такие структуры получаются, если в каждой паре семейств функции $\psi_{2 l-1}^{(m)}=-\psi_{2 l}^{(m)}, \omega_{2 l-1}^{(m)}=\omega_{2 l}^{(m)}$ зависят только от одной переменной:

$$
\begin{gathered}
\psi_{2 l-1}^{(m)}\left(x_{i}\right)=-\psi_{2 l}^{(m)}\left(x_{i}\right) ; \\
\omega_{2 l-1}^{(m)}\left(x_{i}\right)=\omega_{2 l}^{(m)}\left(x_{i}\right)=\omega_{2 l}^{*(m)} / \cos \psi_{2 l}^{(m)}\left(x_{i}\right),
\end{gathered}
$$

где последнее равенство является следствием условия постоянства поперечных сечений волокон (42) (или (41) при $H_{m}-H_{m-1}=$ const); $\omega_{2 l}^{*(m)}=$ const - заданные постоянные интегрирования [14]. Если $H_{m}-H_{m-1}=f_{m}\left(x_{i}\right) \neq c o n s t$, то из (41) в случае пространственного армирования вместо (73) с учетом (72) получим

$$
\omega_{2 l-1}^{(m)}\left(x_{i}\right)=\omega_{2 l}^{(m)}\left(x_{i}\right)=\omega_{2 l}^{*(m)} /\left[f_{m}\left(x_{i}\right) \cos \psi_{2 l}^{(m)}\left(x_{i}\right)\right]\left(i=1 \text { или } i=2,1 \leq l \leq L^{(m)}, 0 \leq m \leq M\right) .
$$

Для таких структур армирования в качестве базисных функций в (68) можно использовать

$$
X_{1 i}\left(x_{1}\right)=\sin \left(\pi i x_{1} / c\right), X_{2 j}\left(x_{2}\right)=\sin \left(\pi j x_{2} / b\right), i, j=1,2, \ldots,
$$

удовлетворяющие условиям (69), (71).

Если некоторые стороны кромки прямоугольной пластины защемлены, а другие - оперты (при выполнении на опертых кромках условий, аналогичных (72)-(74)), то также можно использовать соответствующие балочные функции для задания $X_{1 i}\left(x_{1}\right), X_{2 j}\left(x_{2}\right)$ в (68).

Отметим, что разложение вида (68) при соответствующем выборе функций $X_{1 i}\left(x_{1}\right)$, $X_{2 j}\left(x_{2}\right)$ можно использовать и в случае круглых или кольцевых пластин, а также и для пластин, занимающих в плане область в виде кругового или кольцевого сектора и др. При этом под $x_{1}$ следует понимать полярный радиус, под $x_{2}$ - полярный угол, а уравнение (59) и соответствующие ему граничные условия нужно переписать в полярной системе координат.

В заключение настоящего раздела кратко обсудим возможность использования еще одного обобщенного метода Рунге-Кутта для численного интегрирования задачи динамического изгиба слоисто-волокнистых пластин. Для простоты рассмотрим линейно-упругое деформирование всех фаз композиции. В этом случае $D_{i j} \equiv 0$ и вместо (50) получим линейное уравнение движения параболического типа

$$
w_{, t}(\mathbf{x}, t)+\frac{2}{3 R(\mathbf{x})} \sum_{i=1}^{2} \sum_{j=1}^{2}\left(\sum_{s=1}^{2} \sum_{l=1}^{2} C_{i j s l}(\mathbf{x}) w_{, s l}(\mathbf{x}, t)\right),_{i j}=q(\mathbf{x}, t) \quad\left(t \geq t_{0}, \quad \mathbf{x} \in G\right),
$$


где коэффициенты $C_{i j s l}$ не зависят от $w_{, i j}$ и определены в (25) при $h_{k}^{(m)}=h^{(m)}=H_{m}$, а функция $q$ задана в (54).

Численно проинтегрировать уравнение (76) можно по-прежнему по схеме (55)—(57), где $L$, $D$ суть линейные дифференциальные операторы, в которых $D_{i j} \equiv 0$. Если ввести в рассмотрение вспомогательную функцию $W=\left(w^{n+1}+w^{n}\right) / 2$, то из (55), (56) получим следующую схему:

$$
\begin{aligned}
& w^{n+1}(\mathbf{x})=2 W(\mathbf{x})-w^{n}(\mathbf{x}), v^{n+1}(\mathbf{x})=4\left(W(\mathbf{x})-w^{n}(\mathbf{x})\right) / \tau-v^{n}(\mathbf{x}) ; \\
& \tau^{2} \frac{2}{3} \sum_{i=1}^{2} \sum_{j=1}^{2}\left(\sum_{s=1}^{2} \sum_{l=1}^{2} C_{i j s l}(\mathbf{x}) W,,_{s l}(\mathbf{x}){ }_{, i j}+4 R(\mathbf{x}) W(\mathbf{x})=\tau^{2} p^{n+1 / 2}(\mathbf{x})+2 R(\mathbf{x})\left(\tau v^{n}(\mathbf{x})+2 w^{n}(\mathbf{x})\right),\right.
\end{aligned}
$$

где

$$
p^{n+1 / 2}(\mathbf{x})=\left(p^{n}(\mathbf{x})+p^{n+1}(\mathbf{x})\right) / 2=p\left(\mathbf{x}, t_{n}+\tau / 2\right)+O\left(\tau^{2}\right) .
$$

Равенства (77) формально совпадают с уравнениями одностадийного обобщенного метода Гаусса-Лежандра (метода средней точки), также имеющего второй порядок точности по $\tau$. (Единственное количественное отличие заключается лишь в том, что в методе средней точки $p^{n+1 / 2}(\mathbf{x})=p\left(\mathbf{x}, t_{n}+\tau / 2\right)$. Эта функция отличается от (78) на малую величину $O\left(\tau^{2}\right)$ порядка точности численной схемы.) Следовательно, в линейном случае обобщенные методы средней точки и трапеций формально совпадают.

В $[17,20]$ авторы подробно исследовали применимость обобщенных методов Рунге-Кутта (в частности, метода средней точки) для численного интегрирования задачи поперечных колебаний упругой однородной цилиндрической оболочки, описываемой линейным параболическим уравнением, содержащим, как и (76), производные от прогиба второго порядка по времени и четвертого порядка по пространственной переменной. Предполагая, что из какихто соображений известны собственные функции начально-краевой задачи для однородного уравнения (76) ( $q \equiv 0)$, и дословно повторяя ход рассуждений, приведенный в $[17,20]$, можно доказать спектральную устойчивость схемы (77) при $p^{n+1 / 2} \equiv 0$ и нулевых граничных условиях. (Это доказательство весьма громоздко, поэтому не будем его здесь приводить.) Из спектральной устойчивости следует устойчивость по начальным данным в энергетической норме [21]. Известно [21], что для двухслойных схем (к которым относятся схемы (55), (57), (59) и (77)), применяемых к интегрированию линейных уравнений типа (76), из устойчивости по начальным данным следует устойчивость по правой части. Следовательно, численная схема (77), а значит и схема (55), (59), устойчива при линейно-упругом деформировании фазовых материалов. Аналогично можно доказать устойчивость рассматриваемых схем и при разгрузке всех фаз композиции, когда $C_{i j s l}, D_{i j}$ суть известные функции переменных $x_{1}, x_{2}$ (см. (37)-(40)); при этом известную функцию $\frac{2}{3} \sum_{i=1}^{2} \sum_{j=1}^{2} D_{i j}, i j(\mathbf{x})$ в (50) можно объединить с $p(\mathbf{x}, t)$ и рассматривать как фиктивную составляющую внешней распределенной нагрузки, после чего уравнение (50) редуцируется в (76), для которого схема (55), (59) устойчива.

При наличии пластических зон в фазах композиции, когда $C_{i j l}, D_{i j}$ суть нелинейные операторы от прогиба $w$, устойчивость численной схемы (55)-(63) авторам пока доказать не удалось. Но в пользу устойчивости этой схемы говорит физическая корректность (непротиворечивость) результатов многочисленных расчетов, проведенных авторами, и тот факт, что при переходе от линейно-упругого к упругопластическому деформированию (и наоборот - разгрузка) конструкции тип уравнения движения (50) не изменяется.

Несмотря на формальное сходство методов средней точки и трапеций (77), (78) в случае линейно-упругих колебаний пластины, эти методы существенно различаются при нелинейных (неупругих) колебаниях. В настоящем исследовании для интегрирования системы (53) использовался метод трапеций (55)-(63), а не метод средней точки (77) (хотя, на первый взгляд, второй метод кажется более компактным) по следующим соображениям. Согласно методу трапеций, на $(n+1)$-ом «слое» по времени прогиб $w^{n+1}(\mathbf{x})$ определяется из граничной задачи для уравнения (59), коэффициенты которого $C_{i j s l}, D_{i j}$ (см. (57)) зависят от параметров искривления срединной плоскости пластины $w_{, i j}^{n+1}$, т.е. от состояния фаз композиции (упругого или пласти- 
ческого). Согласно же методу средней точки, прогиб $w^{n+1}$ определяется первым равенством (77) с использованием вспомогательной функции $W$, которую в силу коллокационного свойства этого метода [18], можно рассматривать как прогиб на промежуточном $(n+1 / 2)$-ом «слое» по времени $\left(t_{n+1 / 2}=t_{n}+\tau / 2\right)$. Функция $W$ определяется из граничной задачи для уравнения, формально совпадающего с (59) при замене $w^{n+1}$ на $W$ и при выражении для $P_{n+1}$, совпадающем с правой частью третьего уравнения (77). При этом коэффициенты $C_{i j s}, D_{i j}$ в таком уравнении зависят от $W,_{i j}$, т.е. от состояния фаз композиции (упругих и пластических) на промежуточном $(n+1 / 2)$-ом «слое» по времени. А значит, при вычислении прогиба $w^{n+1}$ не используется информация о состоянии фаз композиции на $(n+1)$-ом «слое» по времени; состояния фаз композиции как бы экстраполируются с промежуточного «слоя» $(n+1 / 2)$ на $(n+1)$-ый «слой». $\mathrm{B}$ силу этого недостатка метода средней точки авторы предпочли ему метод трапеций.

Обсуждение результатов расчетов упругопластической динамики пластин. В качестве примера рассмотрим упругопластический динамический изгиб прямоугольной удлиненной пластины. Предполагается, что внешняя распределенная поперечная нагрузка, закрепление пластины, структуры армирования и толщины слоев не изменяются в продольном направлении. Тогда, пренебрегая локальными торцевыми эффектами, изгиб такой пластины можно считать цилиндрическим и для описания неупругого поведения фазовых материалов даже при развитых пластических деформациях можно обоснованно использовать деформационную теорию пластичности, так как путь деформирования каждой точки конструкции в этом случае является простым [9].

Сориентируем пластину так, чтобы ось $O x_{2}$ совпадала с продольным направлением пластины. Тогда в силу сделанных предположений прогиб $w$ зависит только от времени $t$ и координаты $x_{1}$ в поперечном направлении. Для численного интегрирования соответствующей начально-краевой задачи можно использовать метод (55), (57)-(63), где оператор $D$ редуцируется к виду (см. (57))

$$
D\left(x_{1} ; w^{n}\right)=\frac{2}{3} \frac{d^{2}}{d x_{1}^{2}}\left(C_{1111}\left(x_{1} ; \frac{d^{2} w^{n}\left(x_{1}\right)}{d x_{1}^{2}}\right) \frac{d^{2} w^{n}\left(x_{1}\right)}{d x_{1}^{2}}-D_{11}\left(x_{1} ; \frac{d^{2} w^{n}\left(x_{1}\right)}{d x_{1}^{2}}\right)\right) .
$$

Для численного интегрирования двухточечной граничной задачи, соответствующей уравнению (59), где следует х заменить на $x_{1}$ и учесть (79), можно использовать описанную выше линеаризацию с последующим применением метода матричной прогонки на трехточечном шаблоне с регулярной сеткой. (Более подробно реализацию этого алгоритма см. в [3], так как цилиндрический изгиб кирхгофовской пластины качественно схож с изгибом стержней.) В целом такой метод имеет второй порядок точности по обеим переменным $x_{1}, t$.

Исследуем динамический изгиб изотропных и армированных однослойных и трехслойных прямоугольных удлиненных пластин шириной $l=1 \mathrm{~m}$. Толщина пластин может быть постоянной $\left(2 H(x)=2 H_{*}=\right.$ const $)$ или переменной и определяется формулой

$$
2 H\left(x_{1}\right)=2 \eta H_{*}+\pi \sin \left(\pi x_{1} / l\right)(1-\eta) H_{*} \quad\left(0 \leq x_{1} \leq l, \eta \geq 0\right) .
$$

(При однородном армировании пластин $\left(\omega_{k}=\right.$ const $)$ и задании их толщины в виде (80) расход материала $k$-той фазы композиции будет такой же, как и в пластине постоянной толщины $2 H_{*}$, так как объем пластин одинаков и равен $H_{*} l b$, где $b(l)-$ длина пластины вдоль оси $x_{2}$. При значениях параметра $0 \leq \eta<1$ из (80) следует, что толщина пластины на кромках $x_{1}=0, l$ меньше, чем в центре $\left(x_{1}=l / 2\right)$; при $\eta=1$ получаем пластину постоянной толщины $2 H_{*}$. ) Для пластин постоянной толщины примем $2 H_{*}=0,03$ м. На кромках $x_{1}=0, l$ пластины могут быть шарнирно оперты или защемлены. Внешняя распределенная нагрузка является нагрузкой взрывного типа и затухает по экспоненциальному закону

$$
p\left(x_{1}, t\right)=p(t)=p_{0} \exp (-\alpha t) \quad\left(\alpha=12 \mathrm{c}^{-1}, t \geq 0\right),
$$

где $p_{0}-$ значение нагрузки в начальный момент времени $t=0$, в который конструкция находится в покое, т.е. $w^{0}=0, v^{0}=0$ (см. (47)). Изотропные пластины изготовлены из алюминиевого сплава АДН; армированные пластины изготовлены из того же сплава АДН и усилены одно- 
родно и прямолинейно тремя семействами борных волокон $\left(\psi_{1}=0, \psi_{2}=-\psi_{3} \geq 0, \omega_{2}=\omega_{3}\right)$. Механические характеристики фазовых материалов приведены в табл. 1.

Т а б ли ц а 1

Механические характеристики фазовых материалов [10]

\begin{tabular}{|c|c|c|c|c|r|}
\hline Материал & $E$, ГПа & $v$ & $\sigma_{0,2}$, МПа & $\sigma_{\mathrm{B}}, \mathrm{M \Pi а}$ & $\delta, \%$ \\
\hline Сплав АДН & 71,0 & 0,325 & 100,0 & 150,0 & 6,0 \\
Волокна бора & 416,0 & 0,23 & - & 3150,0 & 0,2 \\
\hline
\end{tabular}

Будем подбирать максимальные значения $p_{0}$ в (81) так, чтобы за характерный период времени $T>0$, в течение которого исследуется процесс колебаний, напряженное состояние в фазах композиции пластины не превышало некоторого предельного состояния. Через $p_{s}$ обозначим $\max p_{0}$, при котором хоть в одной из фаз композиции напряженное состояние достигает предела текучести $\sigma_{0,2}$, но пластичность не развивается (при $p_{0} \leq p_{s}-$ упругие колебания, при $p_{0}>p_{s}-$ упругопластические колебания); через $p_{s s}$ обозначим $\max p_{0}$, при котором хоть в одной из фаз композиции достигается вторичная пластичность, но вторичная пластичность не развивается (при $p_{s}<p_{0} \leq p_{s s}-$ первичная пластичность и разгрузка, при $p_{0}>p_{s s}-$ вторичная пластичность); через $p_{y}$ обозначим значение $p_{0}$, при котором в момент $t_{\max }$ достижения центральным сечением пластины $\left(x_{1}=l / 2\right)$ максимального прогиба напряженное состояние хоть в одной из фаз композиции достигает предела временного сопротивления $\sigma_{\text {в }}$ (начальное разрушение; при $t \leq t_{\max }-$ первичная пластичность, при $t>t_{\max }-$ первичная разгрузка в опасном сечении пластины).

В качестве характерного периода $T$ выберем время, в течение которого нагрузка (81) уменьшается в 1000 раз $(T=-\ln (0,001) / \alpha=0,576 \mathrm{c})$. При $t>T$ внешняя нагрузка ничтожно мала $\left(p(t)<0,001 p_{0}\right)$ и колебания можно считать установившимися, по крайней мере при упругом изгибе.

В расчетах характерный период времени $T$ будем разбивать на 1000 «слоев» $(\tau=T / 1000)$, а по ширине пластины равномерно введем 401 узел.

Исследуем динамическое поведение регулярно армированных по толщине пластин $(M=0)$ со следующими структурами армирования: 1) поперечное армирование $\left(\omega_{1}^{(0)}=0,4\right.$, $\omega_{2}^{(0)}=\omega_{3}^{(0)}=0$ или, что то же самое, $\left.\omega_{2}^{(0)}=\omega_{3}^{(0)}=0,2, \psi_{2}^{(0)}=-\psi_{3}^{(0)}=0, \omega_{1}^{(0)}=0\right)$; 2) перекрестное армирование под углами $\left.\psi_{2}^{(0)}=-\psi_{3}^{(0)}=\pi / 6\left(\omega_{1}^{(0)}=0, \omega_{2}^{(0)}=\omega_{3}^{(0)}=0,2\right) ; 3\right)$ перекрестное армирование под углами $\psi_{2}^{(0)}=-\psi_{3}^{(0)}=\pi / 4\left(\omega_{1}^{(0)}=0, \omega_{2}^{(0)}=\omega_{3}^{(0)}=0,2\right)$; 4) квазиизотропное армирование $\left(\psi_{1}^{(0)}=0, \psi_{2}^{(0)}=-\psi_{3}^{(0)}=\pi / 3, \omega_{1}^{(0)}=\omega_{2}^{(0)}=\omega_{3}^{(0)}=0,4 / 3\right)$. В каждой точке таких пластин суммарная плотность армирования $\sum_{k} \omega_{k}^{(0)}=0,4$ и общий расход арматуры при фиксированной геометрии одинаков.

Для сравнения исследуем динамку трехслойных $(M=1)$ пластин тех же размеров, изготовленных из тех же фазовых материалов, с тем же расходом арматуры, при аналогичных структурах армирования внешних несущих слоев. Внутренний слой является изотропным и изготовлен из сплава АДН; внешние слои изготовлены также из сплава АДН и однородно армированы тремя семействами волокон бора с суммарной плотностью $\sum_{k} \omega_{k}^{(m)}=0,7(m=1,2)$, что, как правило, на практике соответствует максимально допустимой плотности армирования. Чтобы общий расход волокон в трехслойных пластинах был такой же, как в регулярно армированных по толщине конструкциях, рассмотренных выше, толщину внутреннего слоя в трехслойных пластинах нужно задать так: $2 H_{0}=2(0,7-0,4) H / 0,7=6 H / 7$ (где толщина $2 H$ определена в (80)), а плотности армирования соответствующих семейств волокон в несущих слоях нужно увеличить в $7 / 4$ раза. 
В табл. 2 и 3 приведены рассчитанные значения $p_{s}, p_{s s}, p_{y}$ для шарнирно опертых и защемленных пластин постоянной толщины $\left(H=H_{*}\right)$ соответственно, а в табл. $4-$ для шарнирно опертых пластин переменной толщины (80) при значении параметра $\eta=0,5$ (на кромках толщина такой пластины вдвое меньше, чем в случае $H=$ const, а в центре - в $(1+\pi / 2) / 2 \approx 1,29$ раза больше). Структура с номером «0» соответствует изотропной пластине из сплава АДН.

Т а бл и ц а 2

\section{Предельные динамические характеристики шарнирно опертых пластин постоянной толщины $(\eta=1)$}

\begin{tabular}{|c|c|c|c|c|c|c|}
\hline \multirow{2}{*}{$\begin{array}{c}\text { Номер структуры } \\
\text { армирования }\end{array}$} & \multicolumn{3}{|c|}{ Регулярно армированные по толщине } & \multicolumn{3}{|c|}{ Трехслойные пластины } \\
\cline { 2 - 7 } & $p_{s}$, кПа & $p_{s s}$, кПа & $p_{y}$, кПа & $p_{s}$, кПа & $p_{s s}$, кПа & $p_{y}$, кПа \\
\hline 0 & 70,1 & 99,5 & 273,4 & 70,1 & 99,5 & 273,4 \\
1 & 168,5 & 219,4 & 872,3 & 235,4 & 306,7 & 1380,1 \\
2 & 110,8 & 146,8 & 689,8 & 143,1 & 186,5 & 1063,3 \\
3 & 72,5 & 97,2 & 520,0 & 74,2 & 97,9 & 734,9 \\
4 & 88,1 & 117,8 & 401,2 & 100,8 & 132,7 & 557,8 \\
\hline
\end{tabular}

Т а б л и ц а 3

Предельные динамические характеристики жестко защемленных пластин постоянной толщины $(\eta=1)$

\begin{tabular}{|c|c|c|c|c|c|c|}
\hline \multirow{2}{*}{$\begin{array}{c}\text { Номер структуры } \\
\text { армирования }\end{array}$} & \multicolumn{3}{|c|}{ Регулярно армированные по толщине } & \multicolumn{3}{|c|}{ Трехслойные пластины } \\
\cline { 2 - 7 } & $p_{s}$, кПа & $p_{s s}$, кПа & $p_{y}$, кПа & $p_{s}$, кПа & $p_{s s}$, кПа & $p_{y}$, кПа \\
\hline 0 & 111,8 & 152,3 & 434,7 & 111,8 & 152,3 & 434,7 \\
1 & 272,4 & 357,9 & 1371,0 & 381,4 & 504,8 & 2157,5 \\
2 & 174,8 & 235,6 & 1114,0 & 235,1 & 308,3 & 1671,7 \\
3 & 117,3 & 155,5 & 851,0 & 122,1 & 160,3 & 1198,0 \\
4 & 141,1 & 187,8 & 633,6 & 160,3 & 214,5 & 883,6 \\
\hline
\end{tabular}

\section{Предельные динамические характеристики шарнирно} опертых пластин переменной толщины $(\eta=0,5)$

\begin{tabular}{|c|c|c|c|c|c|c|}
\hline \multirow{2}{*}{$\begin{array}{c}\text { Номер структуры } \\
\text { армирования }\end{array}$} & \multicolumn{2}{|c|}{ Регулярно армированные по толщине } & \multicolumn{3}{c|}{ Трехслойные пластины } \\
\cline { 2 - 7 } & $p_{s}$, кПа & $p_{s s}$, кПа & $p_{y}$, кПа & $p_{s}$, кПа & $p_{s s}$, кПа & $p_{y}$, кПа \\
\hline 0 & 103,8 & 173,0 & 407,2 & 103,8 & 173,0 & 407,2 \\
1 & 243,9 & 326,0 & 1270,0 & 361,4 & 471,1 & 2113,9 \\
2 & 163,3 & 219,8 & 1025,0 & 207,0 & 275,8 & 1557,9 \\
3 & 107,6 & 147,7 & 757,7 & 110,9 & 147,5 & 1117,5 \\
4 & 130,2 & 178,1 & 604,6 & 148,5 & 197,9 & 820,0 \\
\hline
\end{tabular}

Значения $p_{s}$, приведенные в табл. 2-4, характеризуются возникновением пластических деформаций в связующем на лицевых поверхностях $(z= \pm H / 2)$ в центральном сечении пластины $x_{1}=l / 2$ (табл. 2), на кромках $x_{1}=0, l$ (табл. 3) и в промежуточных сечениях $x_{1}=x_{*}$, $x_{1}=l-x_{*}, 0<x_{*} \leq l / 2$ (табл. 4); при этом волокна бора имеют значительный (80-процентный и более) запас прочности. Значениям же $p_{s s}$ соответствует появление вторичных пластических деформаций на лицевых поверхностях в связующем в указанных сечениях, а $p_{y}$ характеризуются началом разрушения связующего в изотропных пластинах или упруго-хрупких борных волокон в армированных пластинах (в среднем сечении для шарнирно опертых пластин и на кромках — для защемленных).

Сравнение значений $p_{s}, p_{s s}, p_{y}$, соответствующих изотропным и армированным пластинам, показывает, что за счет армирования конструкции ее несущую способность при динамиче- 
ском изгибе можно увеличить на десятки процентов и даже в разы. Сопоставление тех же значений для армированных пластин со структурами №№ 1 - 3 позволяет проследить за изменением несущей способности конструкции при изменении углов перекрестного армирования от $\psi_{2}=-\psi_{3}=0$ (структура № 1) до $\psi_{2}=-\psi_{3}=\pi / 4$ (структура № 3), откуда следует: варьирование структуры (направления) армирования приводит к существенному (в разы) изменению несущей способности пластины как при упругом, так и упругопластическом изгибах. Поэтому имеет смысл ставить задачи рационального и оптимального армирования тонкостенных конструкций по критериям предельного динамического состояния. Так, из всех рассматриваемых структур наилучшим является армирование в поперечном направлении (вдоль направления $x_{1}$ - структура № 1).

Из табл. 2 - 4 вытекает, что квазиизотропное армирование (структура № 4), часто встречающееся на практике, далеко не всегда обеспечивает высокую несущую способность конструкции при динамическом изгибе, а по критерию $p_{y}$ является худшей из всех рассматриваемых структур армирования.

Сравнение значений $p_{s}$ и $p_{s s}$ показывает, что за счет предварительного нагружения до значений $p_{s s}$ несущую способность изотропной пластины постоянной толщины при шарнирном опирании можно увеличить на $41,9 \%$, при защемлении - на $36,2 \%$, а изотропной пластины переменной толщины - на 66,7 \%. Несущую же способность армированных пластин при таком нагружении можно увеличить на $30-37$ \% (без появления пластичности при повторных нагружениях с интенсивностью $0<p_{0} \leq p_{s s}$ ).

На рисунке изображены кривые, характеризующие колебания центрального сечения $x_{1}=l / 2$ шарнирно опертой трехслойной пластины постоянной толщины с поперечным арми-

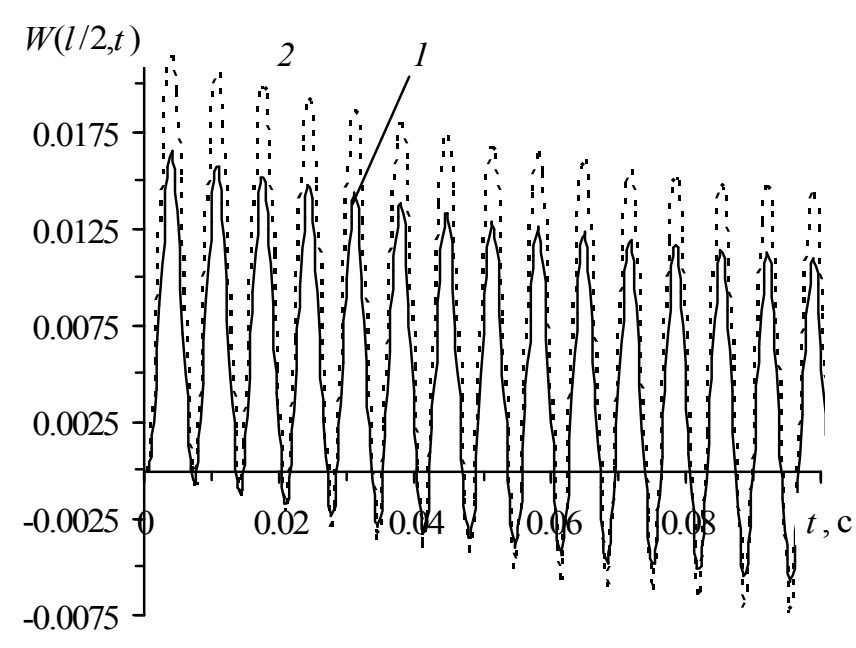

Упругие и упругопластические колебания точек центрального сечения прямоугольной удлиненной пластины

рованием несущих слоев (см. структуру № 1 в табл. 2). Линия 1 соответствует предельным упругим колебаниям конструкции и рассчитана при значении нагрузки $p_{s}=235,4$ кПа; кривая 2 характеризует предельные упругопластические колебания до появления вторичной пластичности и получена при нагрузке $p_{s s}=306,7$ кПа . Сравнение этих линий позволяет получить качественное и количественное представление о приспособляемости пластины при динамическом упругопластическом изгибе. (По оси ординат на рисунке отложен безразмерный прогиб $W=H_{*} w /\left(2 l^{2} \varepsilon_{\mathrm{B} 1}\right)$, где $\varepsilon_{\mathrm{B} 1}=\sigma_{\mathrm{B} 1} / E_{1}>0-$ предельная упругая деформация борных волокон, $\sigma_{\mathrm{в}}, E_{1}-$ предел временного сопротивления и модуль упругости упруго-хрупких борных волокон, см. табл. 1.)

Из сопоставления в каждой табл. 2-4 значений $p_{s}, p_{s s}, p_{y}$ для регулярно армированных по толщине и трехслойных пластин следует: за счет разнесения несущих армированных слоев при том же расходе фазовых материалов несущую способность конструкции при динамическом изгибе можно увеличить от нескольких процентов до 40-66 \%.

Сравнение данных, приведенных в табл. 2 и 3, показывает, что при тех же структурах армирования и одинаковой толщине конструкций несущая способность защемленных пластин на 53-65 \% больше, чем у шарнирно опертых. Это объясняется большей жесткостью защемленных пластин и развитием в них трех пластических зон - в окрестности защемленных кромок и в окрестности центрального сечения $x_{1}=l / 2$; в шарнирно опертых пластинах постоянной толщины развивается лишь одна пластическая зона в окрестности центрального сечения, что приводит к значительному снижению жесткости связующего в этом сечении и более глубокому проникновению пластических деформаций по толщине пластины. 
Сопоставление данных из табл. 2 и 4 позволяет заключить: при одинаковых структурах армирования, расходах фазовых материалов и типах закрепления пластин за счет рационального профилирования (подбора толщины) несущую способность тонкостенной конструкции при динамическом изгибе можно увеличить на $45-75 \%$. Поэтому целесообразно осуществлять рациональное и оптимальное проектирование таких конструкций, отыскивая не только эффективные структуры армирования несущих слоев, но и эффективное распределение слоев в пластине и их толщин.

Отметим, что результаты расчетов, приведенные в табл. $2-4$, получены в предположении о несжимаемости материала связующего (т.е. в упругих зонах коэффициент Пуассона связующего $v^{(m)}$ принимался равным 0,5 , а не 0,325 , как указано в табл. 1$)$. Отказ от учета сжимаемости связующего в упругих зонах приводит к завышению значений $p_{s}, p_{y}$ для изотропных пластин не более чем на $3 \%$, а для армированных пластин - к занижению $p_{s}$ на $9-11 \%$ и завышению $p_{y}$ всего на $1 \%$, однако оказывает существенное влияние на определение величин $p_{s s}$. Как показано в [12], учет сжимаемости в пластической зоне связующего приводит к значительным техническим трудностям (в частности, не позволяет записать коэффициенты $C_{1111}, D_{11}$ в равенстве (79) в явной форме), но при статическом упругопластическом изгибе практически не влияет на результаты расчетов армированных пластин. При учете же сжимаемости в упругой зоне и не учете ее в пластической зоне при переходе через границу от упругой зоны к пластической интенсивность деформаций при цилиндрическом изгибе испытывает скачок на $35 \%$ в сторону увеличения [12]. Этот скачок приводит к искажению информации о накопленных пластических деформациях в связующем и, как следствие этого, о моменте появления вторичной (знакопеременной) пластичности: расчеты показали, что в этом случае для изотропных и армированных пластин всегда $p_{s s}=p_{s}$, т.е. как бы не наблюдается эффект приспособляемости конструкции. Отказ от учета сжимаемости материала связующего как в пластических, так и упругих зонах приводит к непрерывному распределению интенсивности деформаций в конструкции, что позволило отследить эффект приспособляемости пластин при динамическом нагружении $\left(p_{s s} / p_{s}=1,3-1,7\right)$. Значения $p_{s s}$, приведенные в табл. $2-4$ для армированных пластин, по-видимому, занижены на несколько процентов (как и $p_{s}$ ) по сравнению со случаем учета сжимаемости связующего в упругих и пластических зонах.

Выше в качестве характерного периода $T$ было выбрано время, за которое внешняя нагрузка (81) уменьшается по сравнению с начальным значением $p_{0}$ в 1000 раз. Если в качестве $T$ выбрать время 100-кратного уменьшения нагрузки, то из (81) получим $T=0,384 \mathrm{c}$. Расчеты, выполненные при таком $T$, приводят к завышению на $3-5 \%$ значений $p_{s s}$ по сравнению с указанными в табл. 2 -4. (На значения $p_{s}, p_{y}$ выбор времени $T$ не оказывает влияния, необходимо лишь, чтобы время $T$ было больше времени начала полной первичной разгрузки и времени достижения центральным сечением максимального прогиба соответственно.) Если в качестве $T$ выбрать время 20-кратного уменьшения нагрузки $(T=0,250 \mathrm{c})$, то рассчитанные значения $p_{s s}$ будут на десятки процентов превосходить те, что указаны в табл. 2 - 4. Следовательно, при нагрузках взрывного типа (81) в качестве $T$ вполне достаточно выбрать время 1000-кратного уменьшения внешней нагрузки. К концу этого времени колебания становятся установившимися.

Работа выполнена при финансовой поддержке Российского фонда фундаментальных исследований (грант 02 01-00115).

\section{БИБЛИОГРАФИЧЕСКИЙ СПИСОК}

1. Мазалов В.Н., Немировский Ю.В. Динамика тонкостенных пластических конструкций // Проблемы динамики упруго-пластических сред. Сер.: Новое в зарубежной науке. М.: Мир, 1975. Вып. 5. С. 155-247.

2. Комаров К.Л., Немировский Ю.В. Динамика жестко-пластических элементов конструкций. Новосибирск: Наука, 1984. $236 \mathrm{c}$

3. Немировский Ю.В., Янковский А.П. Интегрирование задачи динамического упругопластического изгиба армированных стержней переменного поперечного сечения обобщенными методами Рунге-Кутта // Вычислительные технологии. 2004. Т.9. № 4. С. 77-94.

4. Ананенко Л.А., Комаров К.Л. Динамика неупругих балок. Новосибирск: Наука, 1999. 151 с.

5. Галин М.П. Поперечные колебания балок и плит за пределом упругости под действием импульсных и ударных нагрузок // Изв. АН СССР. ОТН. 1958. № 3. С. $42-50$. 
6. Кошур В.Д., Немировский Ю.В. Континуальные и дискретные модели динамического деформирования элементов конструкций. Новосибирск: Наука, 1990. 200 с.

7. Малинин Н.Н. Прикладная теория пластичности и ползучести. М.: Машиностроение, 1968. 400 с.

8. Соколовский В.В. Теория пластичности. М.: Высш. школа, 1969. 608c.

9. Ильюшин А.А. Пластичность. Основы общей математической теории. М.: Изд-во АН СССР, 1963. 272 с.

10. Композиционные материалы. Справочник. Киев: Наук. думка, 1985. 592 с.

11. Немировский Ю.В. Об упруго-пластическом поведении армированного слоя // Прикладная механика и техническая физика. 1969. № 6. С. 81-89.

12. Янковский А.П. Влияние учета сжимаемости материала на упругопластический изгиб пластин // Труды НГАСУ. 2003. T. 6. № 6. С. 81-97.

13. Москвитин B.B. Циклические нагружения элементов конструкций. М.: Наука, 1981. 344 с.

14. Немировский Ю.В., Янковский А.П. О некоторых особенностях уравнений оболочек, армированных волокнами постоянного поперечного сечения // Механика композитных материалов и конструкций. 1997. Т. 3. № 2. C. $20-40$.

15. Калке Э. Справочник по дифференциальным уравнениям в частных производных первого порядка. М.: Наука, 1966. $260 \mathrm{c}$

16. Немировский Ю.В., Янковский А.П. Численное интегрирование двумерных краевых задач с большими градиентами решения // Вычислительные технологии. 2000. Т. 5. № 4. С. 82-96.

17. Немировский Ю.В., Янковский А.П. Рациональное проектирование армированных конструкций. Новосибирск: Наука, 2002. $488 \mathrm{c}$.

18. Деккер К., Вервер Я. Устойчивость методов Рунге-Кутты для жестких нелинейных дифференциальных уравнений. М.: Мир, 1988. 334 с.

19. Лехницкий С.Г. Анизотропные пластинки. М., Л.: ГИТТЛ. 1947. 355 с.

20. Немировский Ю.В., Янковский А.П. Численное интегрирование динамических задач теории оболочек методами Рунге-Кутты // Численные методы решения задач теории упругости и пластичности: Тр. 16-й Межресп. конф., Новосибирск, 6-8 июля 1999 г. / Под ред. В.М. Фомина. Новосибирск: Изд-во СО РАН, 1999. С. 117-124.

21. Самарский А.А. Теория разностных схем. М.: Наука, 1989. 616 с. 